\title{
FeO Content of Earth's Liquid Core
}

\author{
Monica Pozzo, ${ }^{1}$ Chris Davies, ${ }^{2}$ David Gubbins, ${ }^{2}$ and Dario Alfè ${ }^{1,3, *}$ \\ ${ }^{1}$ Department of Earth Sciences, London Centre for Nanotechnology and \\ Thomas Young Centre at University College London, University College London, \\ Gower Street, London WC1E 6BT, United Kingdom \\ ${ }^{2}$ School of Earth and Environment, University of Leeds, Leeds LS2 9JT, United Kingdom \\ ${ }^{3}$ Dipartimento di Fisica Ettore Pancini, Università di Napoli Federico II, \\ Monte Sant'Angelo, I-80126 Napoli, Italy
}

(Received 1 May 2019; revised manuscript received 19 July 2019; published 24 October 2019)

\begin{abstract}
The standard model of Earth's core evolution has the bulk composition set at formation, with slow cooling beneath a solid mantle providing power for geomagnetic field generation. However, controversy surrounding the incorporation of oxygen, a critical light element, and the rapid cooling rates needed to maintain the early dynamo have called this model into question. The predicted cooling rates imply early core temperatures that far exceed estimates of the lower mantle solidus, suggesting that early core evolution was governed by interaction with a molten lower mantle. Here we develop ab initio techniques to compute the chemical potentials of arbitrary solutes in solution and use them to calculate oxygen partitioning between liquid $\mathrm{Fe}-\mathrm{O}$ metal and silicate melts at the pressure-temperature $(P-T)$ conditions expected for the early core-mantle system. Our distribution coefficients are compatible with those obtained by extrapolating experimental data at lower $P-T$ values and reveal that oxygen strongly partitions into metal at core conditions via an exothermic reaction. Our results suggest that the bulk of Earth's core was undersaturated in oxygen compared to the $\mathrm{FeO}$ content of the magma ocean during the latter stages of its formation, implying the early creation of a stably stratified oxygen-enriched layer below the core-mantle boundary (CMB). FeO partitioning is accompanied by heat release due to the exothermic reaction. If the reaction occurred at the $\mathrm{CMB}$, this heat sink could have significantly reduced the heat flow driving the core convection and magnetic field generation.
\end{abstract}

DOI: 10.1103/PhysRevX.9.041018

Subject Areas: Chemical Physics, Geophysics, Statistical Physics

\section{INTRODUCTION}

It is generally believed that after its formation 4.54 billion years ago, Earth quickly differentiated with a heavy ironbased core sinking to its center during the first 100 million years [1]. As Earth cooled, a solid inner core started to form at the center of the planet, and in the process, it began to expel the primordial—solid incompatible—elements into the liquid. Of particular importance is the expulsion of light elements because Earth uses their gravitational potential energy to drive convection in the liquid outer core, which is presently the main power source for the geodynamo process that generates Earth's magnetic field [2,3]. Recent studies also suggest that gravitational power for the

\footnotetext{
*d.alfe@ucl.ac.uk
}

Published by the American Physical Society under the terms of the Creative Commons Attribution 4.0 International license. Further distribution of this work must maintain attribution to the author(s) and the published article's title, journal citation, and DOI. dynamo can be produced at the top of the core by the precipitation of oxides such as $\mathrm{MgO}[4,5]$ and/or $\mathrm{SiO}_{2}$ [6]. As a result, the dynamics and evolution of Earth's core depend strongly on the nature of its light element inventory.

The density drop between the inner and outer core requires that some elements partition strongly into the liquid on freezing [7], the prime candidate being oxygen [8,9], while other elements (e.g., silicon and sulphur) that partition more equally are needed to match the core mass and seismic velocity. The core's light element inventory, and crucially its oxygen content, is generally thought to have been entirely determined by metal-silicate separation and chemical equilibration at pressure-temperature-composition conditions near the base of an early magma ocean [1], although effects such as entrainment and turbulent mixing could alter this simple picture [10]. In any case, there is significant uncertainty and debate surrounding the amount of $\mathrm{O}$ that can reach the core by this process $[11,12]$. Here we analyze the role of post-core-formation processes in setting the core oxygen content. 
Studies of partitioning between representative lower mantle mineral assemblages and liquid iron suggest that oxygen will transfer into the metal as $\mathrm{FeO}$ [13-18]. The amount of $\mathrm{O}$ that can enter the core from the solid mantle is small because the process is limited by diffusion through the lower mantle chemical boundary layer [18]. However, there is good reason to believe that the lower mantle was once molten, which could allow for substantial mass transfer into the core $[19,20]$. Core evolution models that assess the power requirements for sustaining the geomagnetic field over the last 3.5 Gyr [21] show that past core temperatures inevitably exceeded the lower mantle solidus estimated to be 3500-4200 K [20]. Standard evolution models (those not including precipitation) that incorporate new high core thermal conductivity estimates [22-24] require cooling rates exceeding $100 \mathrm{~K} \mathrm{Gyr}^{-1}$ and predict supersolidus temperatures until the last $0.5-1.0 \mathrm{Ga}$ $[3,20,25]$. Even with lower values of the thermal conductivity $[26,27]$, these models predict supersolidus temperatures for approximately the first 1 Gyr [28] after core formation. Concerns over the inefficiency of sustaining the ancient dynamo with high thermal conductivity led to a new class of models arguing that precipitation of $\mathrm{MgO}$ [4] or $\mathrm{SiO}_{2}$ [6] began shortly after core formation, though these models still predict supersolidus temperatures until the last 1-2 Ga. All evolution models predict that the inner core is $\lesssim 1.5$-Ga old, and so $\mathrm{FeO}$ exchange between a fully molten core and a molten lower mantle should have occurred over much of Earth's history.

The amount of oxygen that can be transferred into the core from a molten lower mantle depends on the thermodynamic equilibrium between silicate melts and liquid metal at core-mantle-boundary (CMB) conditions and the dynamics of mass transfer between the core and the magma ocean. A major challenge is accessing the pressure $(P)$ and temperature $(T)$ conditions of $P=135 \mathrm{GPa}$ and $T \geq 5000 \mathrm{~K}$ relevant for studying core-mantle equilibrium at early times. Despite significant recent progress (e.g., Refs. [29-34]), experiments are presently unable to simultaneously access these $P-T$ conditions, though some have come close (e.g., the values of $P=100 \pm 8 \mathrm{GPa}$ and $T=$ $5700 \pm 500 \mathrm{~K}$ reported in Ref. [29]). However, the majority of experiments have been conducted at $P<80 \mathrm{GPa}$ and $T<5000 \mathrm{~K}$ (Fig. 2), and so the role of temperature and composition at CMB pressures is poorly known.

In this paper, we develop complementary methods to compute the chemical potentials of solutes in solution, and we apply them to the study of equilibrium between Earth's core and the mantle. We focus on partitioning of $\mathrm{FeO} ; \mathrm{MgO}$ and $\mathrm{SiO}_{2}$ will be considered in a future study. The results presented in this work are compared to experiments where possible. We obtain the chemical potentials of iron and oxygen in a silicate melt comprising $50 \% \mathrm{SiO}_{2}, 44 \% \mathrm{MgO}$, and $6 \% \mathrm{FeO}$ and a liquid metal comprising $95 \% \mathrm{Fe}$ and $5 \% \mathrm{O}$ at conditions close to those of the CMB at pressure and temperatures that were typical of early Earth [35]. We find that a significant amount of oxygen can enter the core after its formation.

In Sec. II, we describe the basic theoretical and computational tools used in this work. The methods are based on density-functional theory (DFT) [36,37], and we present three independent statistical mechanics techniques to compute free-energy differences, and from those, the chemical potentials of solutes in solution. In particular, we discuss (i) thermodynamic integration from a reference potential, whereby the potential energy function is converted from that of a reference potential to that of the full DFT system, (ii) inclusion of a solute, whereby one atom of solute is gradually added to the system, and (iii) alchemical transmutation of a solvent into a solute, whereby one atom of solvent is gradually converted into a solute atom. All methods are based on sampling the phase space using molecular dynamics. In Sec. IV, we check the consistency of these alternative methods to obtain the oxygen chemical potential. In Sec. V, we report the results for the distribution coefficient defined as in Ref. [29] as $K_{D}=\left[\left(c_{\mathrm{O}}^{c} c_{\mathrm{Fe}}^{c}\right) /\right.$ $\left.\left(c_{\mathrm{Fe}}^{\mathrm{MO}}\right)\right]$, where $c_{\mathrm{O}}^{c}$ and $c_{\mathrm{Fe}}^{c}$ are, respectively, the molar concentrations of $\mathrm{O}$ and $\mathrm{Fe}$ in the core, and $c_{\mathrm{Fe}}^{\mathrm{MO}}$ is the molar Fe concentration in the magma ocean. Here and throughout the paper, the superscripts $c$ and MO signify "core" and "magma ocean." respectively. Finally, in Sec. VI we discuss the implications of our results for Earth.

\section{THEORY}

\section{A. The chemical potential}

The chemical potential of a solute atom of type $X$ in a solvent made of atoms of type $A$ (with no loss of generality, the discussion also applies to the case where $A=X$ ) is defined as the change of free energy as one atom of $X$ is added to the system. It can be defined as

$$
\mu_{X}\left(p, T, c_{X}\right)=\left(\frac{\partial G}{\partial N_{X}}\right)_{p, T, N_{A}},
$$

where $G$ is the Gibbs free energy, $p$ the pressure, $T$ the temperature, $N_{X}$ the number of $X$ atoms, $N_{A}$ the number of $A$ atoms, and $c_{X}$ the concentration given by $c_{X}=N_{X} / N$, with $N=N_{A}+N_{X}$. The Gibbs free energy of the system is also directly related to the chemical potentials by $G=$ $N_{X} \mu_{X}+N_{A} \mu_{A}$.

The chemical potential can be equivalently defined as

$$
\mu_{X}\left(v, T, c_{X}\right)=\left(\frac{\partial F}{\partial N_{X}}\right)_{V, T, N_{A}},
$$

where $F$ is the Helmholtz free energy, $V$ is the volume of the system, and $v=V / N$ is the average volume per atom.

For finite systems, the two definitions in Eqs. (1) and (2) provide, in general, slightly different chemical potential 
values, because as one atom is added at constant volume the pressure of the system increases. However, one can show that (see the Appendix A)

$$
\mu_{X}\left(p, T, c_{X}\right) \simeq \mu_{X}\left(v, T, c_{X}\right)-\frac{V}{2 B_{T}} \delta p^{2},
$$

where $B_{T}$ is the isothermal bulk modulus of the system, and $\delta p$ is the change of pressure as the atom $X$ is added to the system at constant volume $V$ (see Appendix A). The approximate relation in Eq. (3) is useful for our discussion, because although we need the chemical potential at fixed pressure, we prefer in most cases to work at constant volume, and so we use Eq. (3) to correct the results. We now describe three independent complementary methods to compute $\mu_{X}\left(v, T, c_{X}\right)$. They are all based on the idea that free-energy differences can be computed using thermodynamic integration in which an initial potential energy function is slowly switched to a final potential energy function. The switching is done adiabatically, i.e., in such a way that the system follows a path of states in quasiequilibrium, so that the partition function is sampled in the relevant region of the phase space at each point on the path.

\section{Method 1}

The first method that we describe relies on the calculation of the Helmholtz free energy of the system as the difference from that of a chosen reference system.
Equation (2) then provides a direct route to the evaluation of $\mu_{X}$. The derivative on the rhs can be computed numerically by calculating the Helmholtz free energy of the system with different number of atoms in the system:

$\mu_{X}\left(v, T, c_{X}\right)=\frac{F\left(V, T, N_{X}, N_{A}\right)-F\left(V, T, N_{X}-d N, N_{A}\right)}{d N}$,

where $d N$ is an integer and $v=V /(N-d N / 2)$. In practical calculations it is useful to set $d N$ as large as possible in order to improve on statistics, but of course, this will provide only an accurate estimate of the chemical potential if the Helmholtz free energy is linear in the range $\left(N_{X}-d N, N_{X}\right)$. A slight ambiguity arises in finite systems in the definition of the concentration of the solute $X$, as this changes from $N_{X} /\left(N_{A}+N_{X}\right)$ to $\left(N_{X}-\right.$ $d N) /\left(N_{A}+N_{X}-d N\right)$ for the two terms in the numerator on the rhs of Eq. (4). To remove this ambiguity, we take $c_{X}=\left(N_{X}-d N / 2\right) /\left(N_{A}+N_{X}-d N / 2\right)$, which is the average between the concentrations before and after the $d N$ atoms of type $X$ are added to the system.

In the classical limit (i.e., ignoring nuclei quantum effects), the Helmholtz free energy of a system including $N_{X}$ solute and $N_{A}$ solvent atoms that have access to the whole volume $V$ can be expressed as

$$
F\left(V, T, N_{A}, N_{X}\right)=-k_{B} T \ln \left\{\frac{1}{N_{A} ! N_{X} ! \Lambda_{A}^{3 N_{A}} \Lambda_{X}^{3 N_{X}}} \int_{V} d \mathbf{R} \exp \left[-\beta U\left(N_{A}, N_{X} ; \mathbf{R}\right)\right]\right\}
$$

where $\beta=1 / k_{B} T, k_{B}$ being the Boltzmann constant, and $\Lambda_{A}, \Lambda_{X}$ the thermal wavelengths of $A$ and $X$ given by $\Lambda_{A}=h /\left(2 \pi M_{A} k_{B} T\right)^{1 / 2}$, with $h$ the Plank's constant, $M_{A}$ the atomic mass of $A$, and similarly for $\Lambda_{X}$. The quantity $U\left(N_{A}, N_{X} ; \mathbf{R}\right)$ is the total energy function of the system which depends on the positions of all the atoms indicated by $\mathbf{R}$, and $\int_{V} d \mathbf{R}$ indicates integration over the whole configuration space of the system contained in volume $V$. Separating the terms that do not depend on the potential energy, we rewrite Eq. (5) as

$$
F\left(V, T, N_{A}, N_{X}\right)=-k_{B} T \ln \frac{V^{N_{A}}}{N_{A} ! \Lambda_{A}^{3 N_{A}}}-k_{B} T \ln \frac{V^{N_{X}}}{N_{X} ! \Lambda_{X}^{3 N_{X}}}-k_{B} T \ln \frac{1}{V^{N}} \int_{V} d \mathbf{R} \exp \left[-\beta U\left(N_{A}, N_{X} ; \mathbf{R}\right)\right]
$$

which with the Stirling formula becomes

$$
\begin{aligned}
F\left(V, T, N_{A}, N_{X}\right) & =-k_{B} T N_{A}\left(\ln \frac{V}{N_{A} \Lambda_{A}^{3}}+1\right)-k_{B} T N_{X}\left(\ln \frac{V}{N_{X} \Lambda_{X}^{3}}+1\right)+F^{\mathrm{ex}}\left(V, T, N_{A}, N_{X}\right) \\
& =-k_{B} T N_{A}\left(\ln \frac{V}{N \Lambda_{A}^{3}}-\ln \frac{N_{A}}{N}+1\right)-k_{B} T N_{X}\left(\ln \frac{V}{N \Lambda_{X}^{3}}-\ln \frac{N_{X}}{N}+1\right)+F^{\mathrm{ex}}\left(V, T, N_{A}, N_{X}\right) \\
& =N\left[c_{A} F_{A}^{\mathrm{PG}}(v, T)+c_{X} F_{X}^{\mathrm{PG}}(v, T)+k_{B} T c_{A} \ln c_{A}+k_{B} T c_{X} \ln c_{X}\right]+F^{\mathrm{ex}}\left(V, T, N_{A}, N_{X}\right),
\end{aligned}
$$

where $v=V / N$ is the average volume per atom, $F_{A / X}^{\mathrm{PG}}(v, T)=-k_{B} T\left\{\ln \left[v /\left(\Lambda_{A / X}^{3}\right)\right]+1\right\}$ is the perfect gas Helmholtz free energies per atom of atom $A / X$, and $F^{\mathrm{ex}}\left(V, T, N_{A}, N_{X}\right)=-k_{B} T \ln \left(1 / V^{N}\right) \int_{V} d \mathbf{R} \exp \left[-\beta U\left(N_{A}, N_{X} ; \mathbf{R}\right)\right]$. 
The excess free energy $F^{\mathrm{ex}}\left(V, T, N_{X}, N_{A}\right)$ can be calculated by thermodynamic integration,

$$
\begin{aligned}
F^{\mathrm{ex}}\left(V, T, N_{A}, N_{X}\right)= & F_{\mathrm{ref}}^{\mathrm{ex}}\left(V, T, N_{A}, N_{X}\right) \\
& +\int_{0}^{1} d \lambda\left\langle U\left(N_{A}, N_{X} ; \mathbf{R}\right)\right. \\
& \left.-U_{\mathrm{ref}}\left(N_{A}, N_{X} ; \mathbf{R}\right)\right\rangle_{\lambda}
\end{aligned}
$$

where $U_{\text {ref }}$ is the total energy function of an appropriately chosen reference system, $F_{\text {ref }}^{\mathrm{ex}}$ its excess free energy, and the quantity $\langle\cdot\rangle_{\lambda}$ is the canonical average,

$$
\langle B\rangle_{\lambda}=\frac{\int_{V} d \mathbf{R} B e^{-\beta U_{\lambda}\left(N_{A}, N_{X} ; \mathbf{R}\right)}}{\int_{V} d \mathbf{R} e^{-\beta U_{\lambda}\left(N_{A}, N_{X} ; \mathbf{R}\right)},}
$$

where $U_{\lambda}\left(N_{A}, N_{X} ; \mathbf{R}\right)=\lambda U\left(N_{A}, N_{X} ; \mathbf{R}\right)+(1-\lambda) U_{\text {ref }}\left(N_{A}\right.$, $\left.N_{X} ; \mathbf{R}\right)$, and $B$ is the quantity to be averaged. Note that $F^{\mathrm{ex}}$ in Eq. (8) does not depend on the choice of reference system, provided that no discontinuities are observed in $U_{\lambda}$ as $\lambda$ is increased from zero to 1 .

\section{Method 2}

A second approach to compute the chemical potential of the solute is to add one (or more generally $d N$ ) atom(s) of solute to the system at constant volume and temperature and compute the change of Helmholtz free energy in the process. The main difference with method 1 is that the chemical potential is obtained as the difference of free energies of the systems with $N_{X}$ and $N_{X}-d N$ solute atoms but without explicitly computing the free energies of the two systems. We rewrite Eq. (4) as (see Appendix B)

$$
\begin{aligned}
& \frac{F\left(V, T, N_{A}, N_{X}\right)-F\left(V, T, N_{A}, N_{X}-d N\right)}{d N} \\
& =k_{B} T \ln c_{X}+\mu_{X}^{\mathrm{PG}}(v, T)+\frac{\Delta F}{d N}+\frac{\mathrm{FS}\left(N_{X}, d N\right)}{d N} \\
& =k_{B} T \ln c_{X}+\tilde{\mu}_{X}\left(v, T, c_{X}\right)+\frac{\mathrm{FS}\left(N_{X}, d N\right)}{d N},
\end{aligned}
$$

where the average volume is now $v=V /(N-d N / 2)$, $\mu_{X}^{\mathrm{PG}}(v, T)=F_{X}^{\mathrm{PG}}(v, T)+k_{B} T$ is the perfect gas chemical potential, and $\operatorname{FS}\left(N_{X}, d N\right)$ is a finite-size correction which results from the use of the Stirling formula and the condition $d N \ll N_{X}$. This correction is not necessary for method 1 or method 3 (described below), because in those cases, only the excess free energy is computed with finitesize systems. However, in method 2, we compute part of the free energy by performing thermodynamic integration directly from the perfect gas (see below), and therefore, this correction needs to be taken into account, but we note that with the parameters used in the calculations, this correction is of the order of only approximately $-0.03 \mathrm{eV} /$ atom, and therefore, negligible for our present purposes. Equation (10) defines the excess chemical potential $\tilde{\mu}_{X}\left(v, T, c_{X}\right)$. The quantity $\Delta F$ is calculated as

$\Delta F=\int_{0}^{1} d \lambda\left\langle U\left(N_{A}, N_{X} ; \mathbf{R}\right)-U\left(N_{A}, N_{X}-d N ; \mathbf{R}^{\prime}\right)\right\rangle_{\lambda}$,

where $\quad \mathbf{R}^{\prime}=\left(\mathbf{R}_{1}, \ldots, \mathbf{R}_{N_{A}}, \mathbf{R}_{N_{A}+1}, \ldots, \mathbf{R}_{N_{A}+N_{X}-d N}\right) \quad$ and $\mathbf{R}=\left(\mathbf{R}^{\prime}, \mathbf{R}_{N_{A}+N_{X}-d N+1}, \ldots, \mathbf{R}_{N_{A}+N_{X}}\right)$. The canonical average is generated by the potential $U_{\lambda}\left(N_{A}, N_{X} ; \mathbf{R}\right)=$ $\lambda U\left(N_{A}, N_{X} ; \mathbf{R}\right)+(1-\lambda) U\left(N_{A}, N_{X}-d N ; \mathbf{R}^{\prime}\right)$.

This approach is well defined, but it may be statistically inefficient, because as $\lambda$ approaches zero the forces acting on atoms $\left(N_{A}+N_{X}-d N+1, \ldots, N_{A}+N_{X}\right)$ and the forces on their neighbors due to their presence become vanishingly small (in fact, zero in the limit $\lambda=0$ ). Therefore, the distances between these atoms and their neighbors are no longer limited from below, and close approaches cause large energy fluctuations in the term $U\left(N_{A}, N_{X} ; \mathbf{R}\right)-U\left(N_{A}, N_{X}-d N ; \mathbf{R}^{\prime}\right)$. Indeed, this term diverges for $\lambda=0$. However, since the quantity defined in Eq. (11) is finite, this divergency must be integrable and therefore, the quantity $\langle\cdot\rangle_{\lambda}$ must go to infinity more slowly than $\lambda^{-1}$. This consideration suggests that a practical approach to the calculation of $\Delta F$ is to compute the quantity $\langle\cdot\rangle_{\lambda}$ in Eq. (11) at several values of $\lambda$, including values as close as possible to $\lambda=0$ (but obviously not including the value $\lambda=0$ ) and fit the values with a function of the type $f(\lambda)=a \lambda^{-k}+b$. The value of the integral divided by $d N$ is then $\Delta F / d N=\mu_{X}-\mu_{X}^{\mathrm{PG}}-k_{B} T \ln c_{X}=$ $a /(1-k)+b$.

It is also possible to altogether avoid the divergency at $\lambda=0$ by making a change of variable $\eta^{m}=\lambda$, with $m$ some integer greater than 1. The integral in Eq. (11) becomes

$$
\begin{aligned}
\Delta F^{\mathrm{ex}}= & \int_{0}^{1} m \eta^{m-1} d \eta\left\langle U\left(N_{A}, N_{X} ; \mathbf{R}\right)\right. \\
& \left.-U\left(N_{A}, N_{X}-d N ; \mathbf{R}^{\prime}\right)\right\rangle_{\eta},
\end{aligned}
$$

which does not require the value of the integrand for $\eta=0$.

\section{Method 3}

A third approach is to compute the chemical potential of the solute as the difference from the chemical potential of the solvent [38]. This approach is particularly useful in the low concentration limit, as we explain below. We have

$$
\begin{aligned}
& \mu_{X-A}\left(v, T, c_{X}\right) \\
& =F\left(V, T, N_{A}-1, N_{X}+1\right)-F\left(V, T, N_{A}, N_{X}\right) \\
& =-k_{B} T \ln \left(N_{A} /\left[N_{X}+1\right]\right)-k_{B} T \ln \left(\Lambda_{A}^{3} / \Lambda_{X}^{3}\right) \\
& \quad-k_{B} T \ln \left\{\frac{\int_{V} d \mathbf{R} \exp \left[-\beta U\left(N_{A}-1, N_{X}+1 ; \mathbf{R}\right)\right]}{\int_{V} d \mathbf{R} \exp \left[-\beta U\left(N_{A}, N_{X} ; \mathbf{R}\right)\right]}\right\},
\end{aligned}
$$


which can be expressed as

$$
\begin{aligned}
\mu_{X-A}\left(v, T, c_{X}\right)= & k_{B} T \ln \frac{c_{X}}{1-c_{X}}+3 k_{B} T \ln \frac{\Lambda_{X}}{\Lambda_{A}} \\
& +m\left(V, T, c_{X}\right),
\end{aligned}
$$

where we define

$$
\begin{aligned}
& m\left(V, T, c_{X}\right) \\
& \quad=-k_{B} T \ln \left\{\frac{\int_{V} d \mathbf{R} \exp \left[-\beta U\left(N_{A}-1, N_{X}+1 ; \mathbf{R}\right)\right]}{\int_{V} d \mathbf{R} \exp \left[-\beta U\left(N_{A}, N_{X} ; \mathbf{R}\right)\right]}\right\} .
\end{aligned}
$$

Expanding Eq. (14) to linear order in $c_{X}$, we have

$$
\begin{aligned}
\mu_{X-A}\left(v, T, c_{X}\right) \simeq & k_{B} T \ln c_{X}+k_{B} T c_{X} \\
& +3 k_{B} T \ln \frac{\Lambda_{X}}{\Lambda_{A}}+m\left(V, T, c_{X}\right) .
\end{aligned}
$$

For constant pressure and temperature variations, the chemical potential of the solvent is related to that of the solute by the Gibbs-Duhem equation:

$$
c_{A} d \mu_{A}+c_{X} d \mu_{X}=0 .
$$

To obtain an expression for $\mu_{A}\left(p, T, c_{X}\right)$ in the low concentration limit, we first expand $\tilde{\mu}_{X}\left(p, T, c_{X}\right)$,

$$
\tilde{\mu}_{X}\left(p, T, c_{X}\right)=\mu_{X}^{\dagger}(p, T)+\epsilon(p, T) c_{X}+o\left(c_{X}^{2}\right),
$$

and combining Eqs. (17) and (18), together with $c_{A}=$ $1-c_{X}$, we obtain, to linear order in $c_{X}$,

$$
\mu_{A}\left(p, T, c_{X}\right) \simeq \mu_{A}^{0}(p, T)-k_{B} T c_{X},
$$

where $\mu_{A}^{0}(p, T)$ is the chemical potential of the pure solvent. By summing Eqs. (16) and (19), we can now write the chemical potential of the solute as

$$
\begin{aligned}
\mu_{X}\left(p, T, c_{X}\right) \simeq & k_{B} T \ln c_{X}+\mu_{A}^{0}(p, T)+3 k_{B} T \ln \frac{\Lambda_{X}}{\Lambda_{A}} \\
& +m\left(V, T, c_{X}\right)-\frac{V}{2 B_{T}} \delta p^{2} .
\end{aligned}
$$

The quantity $m\left(V, T, c_{X}\right)$ can be computed using thermodynamic integration techniques by defining a hybrid potential energy function $U_{\lambda}=\lambda U\left(N_{X}+1, N_{A}-1 ; \mathbf{R}\right)+$ $(1-\lambda) U\left(N_{X}, N_{A} ; \mathbf{R}\right)$ and performing the integral

$$
\begin{aligned}
m\left(V, T, c_{X}\right)= & \int_{0}^{1} d \lambda\left\langle U\left(N_{X}+1, N_{A}-1 ; \mathbf{R}\right)\right. \\
& \left.-U\left(N_{X}, N_{A} ; \mathbf{R}\right)\right\rangle_{\lambda},
\end{aligned}
$$

with $\langle\cdot\rangle_{\lambda}$ representing the thermal average operator in the ensemble generated by $U_{\lambda}$ with volume $V$ and temperature $T$. Note that $m\left(V, T, c_{X}\right)$ does not depend on the masses of the solute and the solvent, which are therefore free parameters in the molecular dynamics simulations used to compute thermal averages and can be chosen to be equal. This is useful, because a practical implementation of the method involves performing two simultaneous and synchronous MD simulations, one with $U\left(N_{X}+1, N_{A}-1 ; \mathbf{R}\right)$ and the other with $U\left(N_{X}, N_{A} ; \mathbf{R}\right)$, and then moving the atoms in both systems according to the forces obtained from $\mathbf{f}_{\lambda}(\mathbf{R})=\partial U_{\lambda} / \partial \mathbf{R}$. These forces are equal in both simulations for each corresponding atom, and therefore requiring also the corresponding masses to be equal in order to keep the two trajectories equal.

As noted in Ref. [38], a difficulty in a practical implementation of Eq. (21) is that of statistics, as only one solvent atom is transmuted into a solute. A more efficient procedure is to transmute $d N$ atoms simultaneously and define

$$
\begin{aligned}
W\left(N_{X}, N_{A}, d N\right)= & \frac{1}{d N} \int_{0}^{1} d \lambda\left\langle U\left(N_{X}+d N, N_{A}-d N ; \mathbf{R}\right)\right. \\
& \left.-U\left(N_{X}, N_{A} ; \mathbf{R}\right)\right\rangle_{\lambda} .
\end{aligned}
$$

The quantity $W\left(N_{X}, N_{A}, d N ; V\right)$ is equal to the integral of $m\left(V, T, c_{X}\right)$ over the range of concentrations between $N_{X} / N$ and $\left(N_{X}+d N\right) /(N+d N)$. If $d N$ is sufficiently small compared to $N, m\left(V, T, c_{X}\right)$ can be approximated as depending linearly on $c_{X}$ in the interval $N_{X} / N \leq c_{X} \leq$ $\left(N_{X}+d N\right) /(N+d N)$, and we have $W\left(N_{X}, N_{A}, d N\right)=$ $m\left(V, T, c_{X}\right)$, with $c_{X}=\left(N_{X}+d N / 2\right) /(N+d N / 2)$.

This approach is very efficient computationally, because one changes only a small part of the system to obtain the chemical potential difference between two species. However, for large concentrations, or for the general case in which the chemical potential of the solvent is not known, method 3 is not viable. This is, for example, the case of oxygen in the magma ocean, where we are interested only in the value of its chemical potential and not in those of the other elements. However, the approach of Ref. [38] can still be used to cross-check the other two methods where all of them can be applied, which is what we do in Sec. IV.

\section{B. Heat of reaction}

To understand if the $\mathrm{FeO}$ dissolution reaction is endothermic or exothermic, we calculate the heat of reaction defined as

$$
R_{X}=\mu-T\left(\frac{\partial \mu}{\partial T}\right)_{p, c_{X}} .
$$

Note that $R_{X}$ is completely determined by the excess chemical potential $\tilde{\mu}$, with the configurational term 
$k_{X} T \ln c_{X}$ playing no part, so we can equivalently write $R_{X}=\tilde{\mu}-T[(\partial \tilde{\mu}) /(\partial T)]_{p, c_{X}}$. This is obviously clear also by noting that mixing elements that do not interact chemically (e.g., mixing balls of different color but identical otherwise) cannot result in any production of heat. Since we are adding one $\mathrm{FeO}$ f.u. to the core by removing it from the magma ocean, the total heat absorbed or released is given by $R_{\mathrm{FeO}}^{c}-R_{\mathrm{FeO}}^{\mathrm{MO}}$, where $R_{\mathrm{FeO}}^{c}$ is the heat of reaction as one $\mathrm{FeO}$ f.u. is added to the core, and $R_{\mathrm{FeO}}^{\mathrm{MO}}$ is the analogous quantity for the magma ocean.

Computationally, an efficient procedure to calculate $R_{X}$ is to use its equivalent definition given by the enthalpy change $\Delta H[39]$ :

$$
R_{X}=\Delta H_{X}=\Delta E_{X}+p \Delta V_{X},
$$

where $\Delta E_{X}$ and $\Delta V_{X}$ are the internal energy and the volume change as one formula unit $X$ is added to the system at constant pressure and constant temperature. To improve on the statistics, we compute $\Delta H_{\mathrm{FeO}}^{c}$ and $\Delta H_{\mathrm{FeO}}^{\mathrm{MO}}$ by adding $1,2,3$, and $4 \mathrm{FeO}$ f.u. to the respective liquid and fit the results to a linear function.

\section{TECHNICAL DETAILS}

First-principles simulations are performed using the parameter-free $a b$ initio method DFT. The accuracy of DFT depends on the approximation used for the exchangecorrelation functional. With the generalized-gradientcorrected functional known as PW91 [40] used in the present work, the experimentally observed properties of iron are very accurately reproduced, including highpressure properties such as the $\epsilon$-iron equation of state [41-43], the phonon density of states [44], the equation of state, and the speed of sound on the Hugoniot [45], to name a few. DFT-PW91 is also used to model iron mixed with oxygen, sulphur, and silicon at Earth's core conditions $[22,38,46,47]$, obtaining transport properties in good agreement with experimental data $[23,24,48]$. Recent calculations of liquid-iron mixtures in equilibrium with ferropericlase obtained distribution coefficients in good agreement with experiments [18].

The present calculations are performed using the VASP code [49] with the projector-augmented-wave (PAW) method [50,51]. For Fe, we use a PAW with a Ne core and the $3 s$ electrons also frozen in the core (14 valence electrons). For O, we use a PAW with a He core (six valence electrons). For $\mathrm{Mg}$, we use a PAW with a $\mathrm{He}$ and the $2 s$ orbitals frozen in the core (eight valence electrons), and for $\mathrm{Si}$, a PAW with a Ne core (four valence electrons). Single-particle orbitals are expanded in plane waves with a cutoff of $400 \mathrm{eV}$. The core radii are 1.16, 1.06, 0.8, and $0.8 \AA$, respectively. Electronic levels are occupied according to Fermi-Dirac statistics, with an electronic temperature equal to the ionic temperature. An efficient extrapolation of the charge density is used to speed up the $a b$ initio molecular dynamics simulations [52], which are performed by sampling the BZ with the $\Gamma$ point only. The temperature is controlled with a Nosé thermostat [53], and the time step is set to $1 \mathrm{fs}$. As shown in previous work [18], with these parameters, the chemical potentials are found to be converged to better than $\simeq 50 \mathrm{meV}$. With this accuracy, $\log K_{D}$ is converged to better than 0.08 , which is about 1 order of magnitude better than the typical scattering between experimental data (see Fig. 2).

\section{TEST OF TECHNIQUES}

\section{A. Oxygen in liquid iron}

In Table I, we report the results for the excess chemical potential of oxygen in liquid iron, i.e., the quantity $\tilde{\mu}_{\mathrm{O}}=\mu_{\mathrm{O}}-k_{B} T \ln c_{\mathrm{O}}$, where $c_{\mathrm{O}}$ is the oxygen concentration, obtained using method 1 and compared with calculations performed using method 3 . The use of method 3 is possible because the concentrations of oxygen are low, and we know the chemical potential of pure Fe [45]. The calculations are performed with $T=4300 \mathrm{~K}$, and we use $N_{\mathrm{Fe}}=149$. For method 1 , the three different values reported in Table I are computed using $N_{\mathrm{O}}=8, d N=4$; $N_{\mathrm{O}}=6, d N=2$, and $N_{\mathrm{O}}=8, d N=2$, giving average pressures of $130.1,127.8$, and $132.6 \mathrm{GPa}$, respectively. The pressures are different because the volume of the simulation cell is always the same, but the number of atoms is different. They are given as the average pressure between the two systems with $N_{\mathrm{Fe}}+N_{\mathrm{O}}$ and $N_{\mathrm{Fe}}+N_{\mathrm{O}}-d N$ atoms, because one can think of removing $d N$ atoms to the $N_{\mathrm{Fe}}+N_{\mathrm{O}}$ system just as well as adding $d N$ atoms to the $N_{\mathrm{Fe}}+N_{\mathrm{O}}-d N$ system. The calculations with method 3 are performed with $N_{\mathrm{O}}=8, d N=8$ at a pressure $p=$ 133.5 $\mathrm{GPa}$ and corrected to the required pressure using $d \tilde{\mu}_{\mathrm{O}} / d p=v_{\mathrm{O}}$, with $v_{\mathrm{O}}=5.4 \AA^{3}$ the oxygen partial volume. The agreement between the two sets of results is extremely good when considering their statistical

TABLE I. Excess chemical potential of oxygen in liquid iron at $T=4300 \mathrm{~K}$ and various pressures and concentrations calculated using method 1 and compared to data obtained with method 3 . All systems contain $149 \mathrm{Fe}$ atoms, and the number of $\mathrm{O}$ atoms together with $d N$ is reported in each case. For method 3, only one calculation with $N_{\mathrm{O}}=8, d N=8$ is performed at a pressure $p=133.5 \mathrm{GPa}$, and the values reported in the table are corrected using $d \tilde{\mu}_{\mathrm{O}} / d p=v_{\mathrm{O}}=5.4 \AA^{3}$ (see text).

\begin{tabular}{llll}
\hline \hline & \multicolumn{3}{c}{$\left(p, c_{\mathrm{O}}, N_{\mathrm{O}}, d N\right)(\mathrm{GPa}, \mathrm{mol} \%)$} \\
\cline { 2 - 4 }$\tilde{\mu}_{\mathrm{O}}(\mathrm{eV} / \mathrm{atom})$ & $3.2,6,2)$ & $3.9,8,4)$ & $4.5,8,2)$ \\
\hline Method 3 & $-4.33(6)$ & $-4.26(6)$ & $-4.17(6)$ \\
Method 1 & $-4.26(6)$ & $-4.19(5)$ & $-4.11(8)$ \\
\hline \hline
\end{tabular}


TABLE II. Excess chemical potential of oxygen in liquid iron at $T=4300 \mathrm{~K}$ calculated using method 2 and compared to the data obtained with method 3 . The system contains $149 \mathrm{Fe}$ atoms and eight $\mathrm{O}$ atoms, and we use $d N=8$ (see text).

\begin{tabular}{lc}
\hline \hline & $\left(p, c_{\mathrm{O}}, N_{\mathrm{O}}, d N\right)(\mathrm{GPa}, \mathrm{mol} \%)$ \\
\cline { 2 - 2 }$\tilde{\mu}_{\mathrm{O}}(\mathrm{eV} /$ atom $)$ & $(112.8,2.6,8,8)$ \\
\hline Method 3 & $-4.80(6)$ \\
Method 2 & $-4.81(6)$ \\
\hline \hline
\end{tabular}

errors, which in turn, are small enough for our present purposes.

In Table II, we report the results for $\tilde{\mu}_{\mathrm{O}}$ using method 2 and compare them again to those obtained using method 3. For method 2, to compute the integral in Eq. (11) we perform calculations at nine values of $\lambda$ ranging between 0.0075 and 1.0. The values of the integrand are displayed in Fig. 1. The calculations are performed with $T=4300 \mathrm{~K}$ and $p=112.8 \mathrm{GPa}$, using $N_{\mathrm{Fe}}=149, N_{\mathrm{O}}=8, d N=8$. Once again, the agreement between the results obtained with method 2 and method 3 is extremely good.

\section{RESULTS}

\section{A. Equilibrium between Earth's core and the magma ocean}

The tests of the previous section lend confidence to both method 1 and method 2, and we are ready to extend the calculations to the magma ocean. We consider the chemical potential of $\mathrm{Fe}$ and $\mathrm{O}$ together, rather than $\mathrm{O}$ in isolation, because it is likely that any movement of oxygen between the $\mathrm{MO}$ and the liquid core is accompanied by Fe for charge balance reasons. In fact, oxygen could also be moved as $\mathrm{MgO}$ and/or $\mathrm{SiO}_{2}$, but for the purpose of this paper,

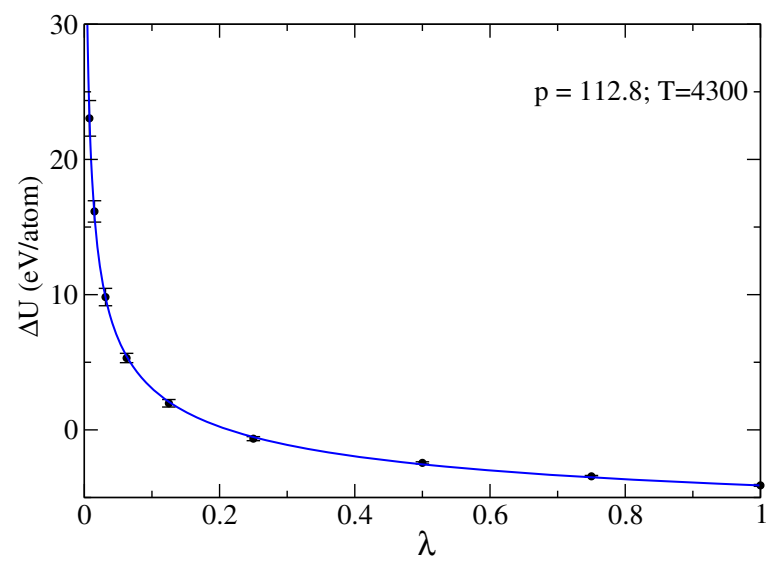

FIG. 1. The integrand of Eq. (11) evaluated at several values of $\lambda$ for the FeO case reported in Table II. The data are fitted to the function $f(\lambda)=a \lambda^{-k}+b$ (blue line), with $k=0.37, a=5.34$, $b=-9.45$.
TABLE III. Excess chemical potential of FeO, $\tilde{\mu}_{\mathrm{FeO}}=\mu_{\mathrm{FeO}}-$ $k_{B} T \ln c_{\mathrm{Fe}}-k_{B} T \ln c_{\mathrm{O}}$, in liquid iron and liquid $\mathrm{Mg}_{28} \mathrm{Fe}_{3} \mathrm{Si}_{32} \mathrm{O}_{95}$, at $T=5500 \mathrm{~K}$ calculated using method 1 [method 2]. For comparison, we also report the value in solid $\mathrm{Mg}_{63} \mathrm{FeO}_{64}$.

\begin{tabular}{llc}
\hline \hline & \multicolumn{2}{c}{$p(\mathrm{GPa})$} \\
\cline { 2 - 3 }$\tilde{\mu}_{\mathrm{FeO}}(\mathrm{eV} /$ atom $)$ & \multicolumn{1}{c}{128.4} & 122.5 \\
\hline Liquid $\mathrm{Mg}_{28} \mathrm{Fe}_{3} \mathrm{Si}_{32} \mathrm{O}_{95}$ & & $-9.76(0.17)$ \\
& & {$[-9.79(0.07)]$} \\
Liquid $\mathrm{Fe}_{148} \mathrm{O}_{7}$ & $-10.46(0.04)$ & \\
Solid $\mathrm{Mg}_{63} \mathrm{FeO}_{64}$ & {$[-10.46(0.07)]$} & $-9.55(0.05)$ \\
\hline \hline
\end{tabular}

we consider only FeO. In Table III, we report the values of the excess chemical potential of $\mathrm{FeO}$ both in a liquid-ironoxygen mixture with $149 \mathrm{Fe}$ and eight $\mathrm{O}$ atoms, and in the $\mathrm{MO}$, modeled as a liquid mixture with a starting composition of $28 \mathrm{Mg}$, four $\mathrm{Fe}, 32 \mathrm{Si}$, and $96 \mathrm{O}$ atoms. To compute the chemical potential with the techniques described in the previous sections, we remove two $\mathrm{FeO}$ units $(d N=2)$ both in the liquid-iron mixture and in the liquid-silicate mixtures. The pressures reported in Table III are the average of the pressures of the systems before and after the removal of the two $\mathrm{FeO}$ molecules. For comparison, we also report the excess chemical potential of $\mathrm{FeO}$ in solid $\mathrm{Mg}_{63} \mathrm{FeO}_{64}$, as computed in Ref. [18]. As expected, the chemical potential is lower in the liquid mixture representing the MO than in solid ferropericlase, but it is still significantly higher than that in the liquid-iron mixture, which will cause strong partitioning of $\mathrm{FeO}$ into the core.

The calculations performed with method 1 are very inefficient because we used a reference potential that is not optimal for liquid $\mathrm{Mg}_{28} \mathrm{Fe}_{3} \mathrm{Si}_{32} \mathrm{O}_{95}$, and so we perform them only at one thermodynamic state to cross-check the results obtained with method 2. All other results are obtained with method 2 only.

Chemical equilibrium is reached when the chemical potential of all species is equal in the two phases. Since we are interested in $\mathrm{FeO}$, we consider the sum of the chemical potentials of Fe and $\mathrm{O}, \mu_{\mathrm{FeO}}^{c}=\mu_{\mathrm{Fe}}^{c}+\mu_{\mathrm{O}}^{c}$ and $\mu_{\mathrm{FeO}}^{\mathrm{MO}}=\mu_{\mathrm{Fe}}^{\mathrm{MO}}+\mu_{\mathrm{O}}^{\mathrm{MO}}$, rather than the two in isolation, that is

$$
\mu_{\mathrm{FeO}}^{c}\left(p, T, c_{\mathrm{O}}^{c}, c_{\mathrm{Fe}}^{c}\right)=\mu_{\mathrm{FeO}}^{\mathrm{MO}}\left(p, T, c_{\mathrm{O}}^{\mathrm{MO}}, c_{\mathrm{Fe}}^{\mathrm{MO}}\right),
$$

where $c_{\mathrm{O}}^{c}$ and $c_{\mathrm{Fe}}^{c}$ are the concentrations of $\mathrm{O}$ and $\mathrm{Fe}$ in the core and similarly for those in the MO. To obtain the relation that governs the partition, we rewrite the chemical potential by separating the configurational parts, and so Eq. (25) becomes

$$
\begin{aligned}
& k_{B} T \ln c_{\mathrm{O}}^{c}+k_{B} T \ln c_{\mathrm{Fe}}^{c}+\tilde{\mu}_{\mathrm{FeO}}^{c}\left(p, T, c_{\mathrm{O}}^{c}, c_{\mathrm{Fe}}^{c}\right) \\
& \quad=k_{B} T \ln c_{\mathrm{O}}^{\mathrm{MO}}+k_{B} T \ln c_{\mathrm{Fe}}^{\mathrm{MO}}+\tilde{\mu}_{\mathrm{FeO}}^{\mathrm{MO}}\left(p, T, c_{\mathrm{O}}^{\mathrm{MO}}, c_{\mathrm{Fe}}^{\mathrm{MO}}\right),
\end{aligned}
$$



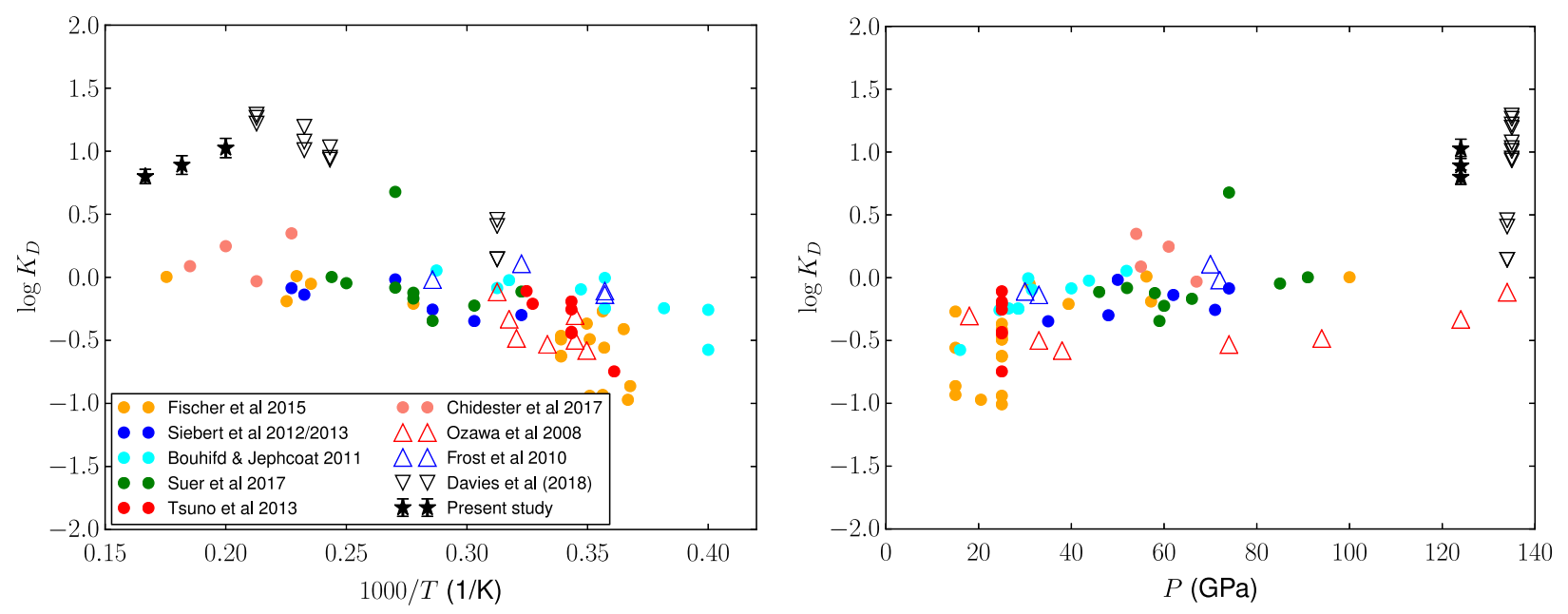

FIG. 2. Comparison of present results with published work. Panels show values of the distribution coefficient $K_{D}$ plotted against inverse temperature (left) and pressure (right) for our data together with other experimental studies of solid-silicate-liquid-metal partitioning (open symbols) and silicate-melt-liquid-metal partitioning (closed symbols).

from which we get the distribution coefficient:

$$
K_{D}=\frac{c_{\mathrm{O}}^{c} c_{\mathrm{Fe}}^{c}}{c_{\mathrm{Fe}}^{\mathrm{MO}}}=c_{\mathrm{O}}^{\mathrm{MO}} \exp \left(-\frac{\tilde{\mu}_{\mathrm{FeO}}^{c}\left(p, T, c_{\mathrm{O}}^{c}, c_{\mathrm{Fe}}^{c}\right)-\tilde{\mu}_{\mathrm{FeO}}^{\mathrm{MO}}\left(p, T, c_{\mathrm{O}}^{\mathrm{MO}}, c_{\mathrm{Fe}}^{\mathrm{MO}}\right)}{k_{B} T}\right)=c_{\mathrm{O}}^{\mathrm{MO}} \exp \left(-\frac{\delta \tilde{\mu}_{\mathrm{FeO}}}{k_{B} T}\right) .
$$

One important difference in the relation above compared to the partition with solid ferropericlase is that in the latter we do not consider the configurational term due to the oxygen concentration, because in the ferropericlase lattice there is only one possible arrangement of the oxygen atoms, and so there is no associated entropy. However, in the MO the oxygen atoms move around freely, and therefore, there will be a contribution to the chemical potential given by $k_{B} T \ln c_{\mathrm{O}}^{\mathrm{MO}} \sim k_{B} T \ln 0.6$. For the same excess chemical potential difference, this term reduces the distribution coefficient compared to that with solid ferropericlase.

To estimate the value of the distribution coefficient, we need the excess chemical potentials at the same pressure, which we can estimate by using $\partial \tilde{\mu}_{\mathrm{FeO}}^{\mathrm{MO}} / \partial p=$ $v_{\mathrm{FeO}}^{\mathrm{MO}} \sim 0.096(1) \mathrm{eV} / \mathrm{GPa} \quad$ and $\partial \tilde{\mu}_{\mathrm{FeO}}^{c} / \partial p=v_{\mathrm{FeO}}^{c} \sim$ $0.091(1) \mathrm{eV} / \mathrm{GPa}$. These two partial volumes are calculated by adding one $\mathrm{FeO}$ molecule to the system at constant pressure and temperature and measuring the change of volume. Therefore, at $124 \mathrm{GPa}$ and $5500 \mathrm{~K}$, the excess chemical potential difference between the core and the mantle is $\delta \tilde{\mu}_{\mathrm{FeO}}(5500)=-1.21(8) \mathrm{eV}$, and we have $K_{D}=$ $7.8_{-1.2}^{+1.5}$. We then perform additional calculations at $T=5000 \mathrm{~K}$ and $T=6000 \mathrm{~K}$, obtaining $\delta \tilde{\mu}_{\mathrm{FeO}}(5000)=$ $-1.24(8)$ and $\delta \tilde{\mu}_{\mathrm{FeO}}(6000)=-1.22(7) \mathrm{eV}$, giving $K_{D}=$ $10.6_{-2.3}^{+2.6}$ and $K_{D}=6.3_{-1.1}^{+1.3}$. Noting that $\partial \delta \tilde{\mu}_{\mathrm{FeO}} / \partial p=$ $-0.005(2) \mathrm{eV} / \mathrm{GPa}$, the dependence of $K_{D}$ with pressure can be estimated from $\partial K_{D} / \partial p=-K_{D} / k_{B} T \times$ $\partial\left(\delta \tilde{\mu}_{\mathrm{FeO}}\right) / \partial p=0.07(2) \mathrm{GPa}^{-1}$.
Figure 2 compares our results to a number of recent studies of partitioning between liquid metal and silicate melts at different $P-T$ conditions [29-34]. The value at $T=5500 \mathrm{~K} K_{D}=7.8_{-1.2}^{+1.5}$ is comparable to the value of $K_{D}=4.76$ at $P=74 \mathrm{GPa}$ and $T=3700 \mathrm{~K}$ reported in Ref. [32] and slightly higher than the values reported in Refs. [29,34] at similar temperatures and lower pressures. The differences are likely due to variations in the starting compositions and oxygen concentrations in both silicate and metal phases, which are known to strongly influence oxygen partitioning $[15,29,54]$, while uncertainties on the experimental $P-T$ conditions will also contribute. These and other factors produce a scatter of 0.5-1 log units in $K_{D}$ across much of the accessible temperature range, which is consistent with the variation observed at high temperature $(>5000 \mathrm{~K})$ in Fig. 2. In view of the significant technical challenges associated with conducting both experiments and simulations at such extreme conditions, the impressive level of agreement in Fig. 2 demonstrates the mutual consistency of these two fundamentally different yet complementary approaches.

Figure 2 shows that, once pressure and temperature effects are taken into account, the results for partitioning between liquid metal and both solid and liquid silicates display similar behavior across the available $P-T$ range. Values of $K_{D}$ at $T>4000 \mathrm{~K}$ for the solid are higher than values obtained from liquid-metal-silicate melt partitioning at similar temperature, which has been found in previous 
TABLE IV. Temperature $T$, excess chemical potential difference of $\mathrm{FeO}$ between the core and the magma ocean $\tilde{\mu}_{\mathrm{FeO}}^{c}-\tilde{\mu}_{\mathrm{FeO}}^{\mathrm{MO}}$ with error given in brackets, heat of reaction difference $R_{\mathrm{FeO}}^{c}-$ $R_{\mathrm{FeO}}^{\mathrm{MO}}$ as one FeO unit is transferred from the magma ocean to the core, distribution coefficient $K_{D}=\left[\left(c_{\mathrm{O}}^{c} c_{\mathrm{Fe}}^{c}\right) / c_{\mathrm{Fe}}^{\mathrm{MO}}\right]$ and partition coefficient $P=K_{D} / c_{\mathrm{Fe}}^{c}$. All calculated values refer to a pressure of $124 \mathrm{GPa}$ for a silicate melt comprising $50 \% \mathrm{SiO}_{2}, 44 \% \mathrm{MgO}$, and $6 \% \mathrm{FeO}$ and a liquid metal comprising $95 \% \mathrm{Fe}$ and $5 \% \mathrm{O}$.

\begin{tabular}{cccrr}
\hline \hline$T(\mathrm{~K})$ & $\tilde{\mu}_{\mathrm{FeO}}^{c}-\tilde{\mu}_{\mathrm{FeO}}^{\mathrm{MO}}(\mathrm{eV})$ & $R_{\mathrm{FeO}}^{c}-R_{\mathrm{FeO}}^{\mathrm{MO}}(\mathrm{eV})$ & \multicolumn{1}{c}{$K_{D}$} & \multicolumn{1}{c}{$P$} \\
\hline 5000 & $-1.24(0.08)$ & $\ldots$ & 10.6 & 11.2 \\
5500 & $-1.21(0.08)$ & -2.5 & 7.8 & 8.2 \\
6000 & $-1.22(0.07)$ & $\cdots$ & 6.3 & 6.6 \\
\hline \hline
\end{tabular}

studies $[20,35,55]$. This is expected from our calculations because of the configurational entropy contribution due to oxygen in the silicate melt (not present in ferropericlase where the oxygen atoms are fixed in their lattice positions), which reduces the liquid-metal-liquid-silicate partition coefficient by a factor of $c_{\mathrm{O}}^{\mathrm{MO}}$ compared to the liquidmetal-ferropericlase value.

The results are reported in Table IV together with a value of the heat of reaction, which is negative, signaling that the reaction of dissolution of $\mathrm{FeO}$ from the $\mathrm{MO}$ to the core is exothermic. This is in contrast with the heat of reaction from solid ferropericlase to liquid-iron mixtures, which is found to be positive [18], giving an endothermic reaction. These results have profound implications for the early history of Earth, as additional chemical energy would have been provided to the core as it acquired its oxygen inventory.

\section{DISCUSSION}

We use our new $\mathrm{FeO}$ partitioning data to estimate the equilibrium conditions between silicate melts and liquid metal in early Earth. Core formation models often assume that the magma ocean froze from the bottom up, with chemical equilibration occurring at the $P-T$ conditions where metal ponds on top of the solid before sinking to the core (e.g., Refs. [11,12]). Another possibility is that the magma ocean froze outwards from the midmantle [56], in which case, partitioning would occur at the CMB. Core formation probably involved multiple impacts between small bodies and proto-Earth that added material of varying composition and produced magma oceans at different pressuretemperature-composition conditions [11]. These conditions are difficult to constrain because they depend on the detailed dynamics of the accretion and differentiation processes, which are still poorly understood. Here we focus on conditions that prevail following the last impact and consider a range of plausible magma-ocean conditions and silicate-melt compositions in order to estimate the oxygen content of material added in the final stages of core formation.

The temperature for equilibration in the latter stages of core formation depends on the manner in which the magma ocean froze. For bottom-up freezing, the relevant temperature is between the peridotite liquidus and solidus, which could be between 3000 and $5000 \mathrm{~K}$ depending on the pressure (depth) of equilibration [12,57]. For middleout crystallization, the basal magma ocean is expected to remain close to the liquidus [56], in which case, the relevant temperature is towards the upper end of the range quoted above. Nevertheless, it is possible that some material could have equilibrated at even higher temperatures [4]. We therefore consider the range $T=5000-6000 \mathrm{~K}$, noting that our results (Fig. 2) suggest lower temperatures will lead to stronger partitioning. The pressure effect on $K_{D}$ is small (Fig. 2) and is neglected here; changes in $P$ therefore affect the results only insofar as they affect the temperature.

We consider a range of estimates for the molar concentrations of iron and oxygen in the silicate melt $c_{\mathrm{Fe}}^{\mathrm{MO}}$ and $c_{\mathrm{O}}^{\mathrm{MO}}$, respectively. This ignores the potential for partitioning of $\mathrm{MgO}$ and/or $\mathrm{SiO}_{2}$, which are not calculated here and will be the subject of future study, though we note that our results for the solid [18] suggest that the presence of $\mathrm{Si}$ does not strongly affect oxygen partitioning. Reasonable bounds on $c_{\mathrm{Fe}}^{\mathrm{MO}}$ and $c_{\mathrm{O}}^{\mathrm{MO}}$ can be obtained by considering the composition of the present lower mantle, which is thought to consist mainly of $70 \%-80 \%(\mathrm{Mg}, \mathrm{Fe}) \mathrm{SiO}_{3}$ bridgmanite and $15 \%-20 \%(\mathrm{Mg}, \mathrm{Fe}) \mathrm{O}$ ferropericlase [58]. We consider a lower value of $c_{\mathrm{O}}^{\mathrm{MO}}=10 \%$, which is probably too low given the abundance of O-bearing minerals in the lower mantle, and an upper value of $c_{\mathrm{O}}^{\mathrm{MO}}=65 \%$ corresponding to almost pure $\mathrm{SiO}_{2}$. We consider $c_{\mathrm{Fe}}^{\mathrm{MO}}=1 \%-10 \%$, slightly smaller than estimates of $10 \%-20 \%$ in previous work $[35,56]$. Higher values of $c_{\mathrm{O}}^{\mathrm{MO}}$ or $c_{\mathrm{Fe}}^{\mathrm{MO}}$ increase the predicted core oxygen concentration which, as we show below, does not affect the conclusions.

The liquid core is assumed to be composed of iron and oxygen, and so Eq. (27) can be written as $c_{\mathrm{O}}^{c}\left(1-c_{\mathrm{O}}^{c}\right)=$ $c_{\mathrm{Fe}}^{\mathrm{MO}} c_{\mathrm{O}}^{\mathrm{MO}} \exp \left\{-\left[\left(\delta \tilde{\mu}_{\mathrm{FeO}}\right) /\left(k_{B} T\right)\right]\right\}$. Varying $c_{\mathrm{Fe}}^{\mathrm{MO}}, c_{\mathrm{O}}^{\mathrm{MO}}$, and $T$ therefore provides a range of estimates for the equilibrium concentration of liquid metal $c_{\mathrm{O}}^{c}$. At each $T$, we use the corresponding value of $\delta \tilde{\mu}_{\mathrm{FeO}}$, assuming that the effect of composition on $\delta \tilde{\mu}_{\mathrm{FeO}}$ is small.

Figure 3 shows $c_{\mathrm{O}}^{c}$ plotted against $c_{\mathrm{O}}^{\mathrm{MO}}$ for different values of $T$ and $c_{\mathrm{Fe}}^{\mathrm{MO}}$. Unless there is very little iron in the $\mathrm{MO}$ (approximately 1\%), $c_{\mathrm{O}}^{c}$ equals or exceeds the present bulk core oxygen concentration. The main result is therefore that abundant oxygen should partition into the core at high temperatures. However, it is also clear that the oxygen added during the latter stages of core formation cannot be uniformly mixed into the bulk core, otherwise the present-day core oxygen concentration would be higher. The explanation is that this oxygen cannot be mixed to greater depths by convection, and instead remains near the CMB $[15,18,19,59]$ creating a layer of stable stratification. This is consistent with our $K_{D}$ values, which increase with decreasing temperature; as the magma ocean cools 


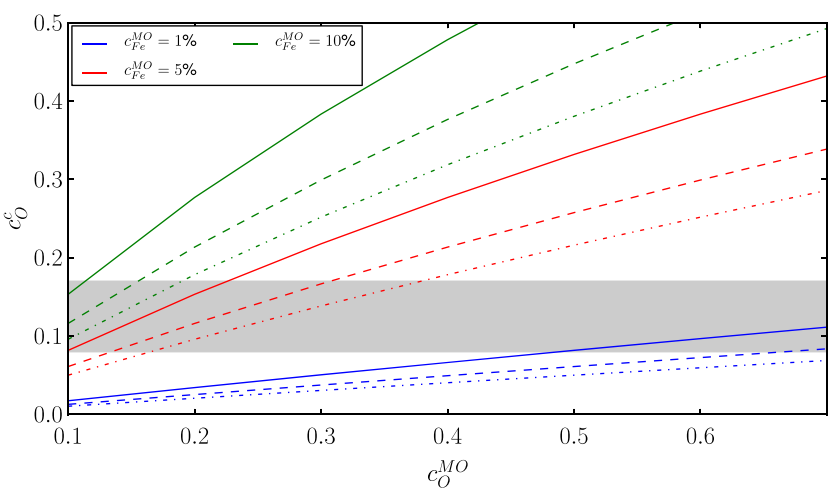

FIG. 3. Predicted equilibrium concentration of oxygen in liquid metal $c_{\mathrm{O}}^{c}$ as a function of oxygen and iron concentrations in silicate melt $c_{\mathrm{O}}^{\mathrm{MO}}$ and $c_{\mathrm{Fe}}^{\mathrm{MO}}$, respectively. Line styles denote different temperatures: $T=5000 \mathrm{~K}$ (solid), $5500 \mathrm{~K}$ (dashed), and $5000 \mathrm{~K}$ (dot-dashed). The gray shaded region highlights the range of oxygen concentrations estimated for the present-day core [25].

following the final giant impact, more oxygen will partition into the core.

Stably stratified layers strongly suppress radial fluid motions (at least in the absence of significant topography on the core-mantle boundary [60]) and support hydromagnetic wave motions that do not arise in convecting regions $[61,62]$. The existence of a stably stratified layer therefore has significant implications for reconciling observed variations of Earth's magnetic field with motions inside the core $[3,63]$. Our results suggest that such a stable stratification would have formed early in Earth's history, directly following the last impact that could have erased any previous stratification [64]. The mechanism is fundamentally different from the primordial compositional stratification that could arise due to merging between a light-element-rich impactor and Earth's core [65], and both effects could possibly reinforce to create a thick and strongly stable region at the top of the core.

If $\mathrm{FeO}$ exchange occurs at the $\mathrm{CMB}$, the release of heat through the exothermic reaction will affect the operation of the dynamo. The reaction does not produce entropy to power the dynamo because heat is both generated and lost at the $\mathrm{CMB}$; the thermodynamic efficiency factor of the process is zero [see Eq. (40) in Ref. [66] ]. However, it does affect the dynamo indirectly by reducing the heat that is extracted from the core at the CMB. The heat sink $Q_{h}$ due to the exothermic reaction can be written [66] $Q_{h}=\left\langle R_{h}\right\rangle\left|I_{\mathrm{O}}\right|$, where $I_{\mathrm{O}}=4 \pi r_{c}^{2} \rho D \Delta c / \delta$ is the mass flux of $\mathrm{O}$ into the core, and $\left\langle R_{h}\right\rangle=R_{\mathrm{O}}^{c}-R_{\mathrm{O}}^{\mathrm{MO}}$. Here, $r_{c}=$ $3480 \mathrm{~km}$ is the CMB radius, $\rho \sim 5000 \mathrm{~kg} \mathrm{~m}^{-3}$ is the lower mantle density, $D \sim 10^{-9} \mathrm{~m}^{2} \mathrm{~s}^{-1}$ is the oxygen-diffusion coefficient, and $\Delta c$ is the change in $\mathrm{O}$ mass fraction across the lower mantle chemical boundary layer of thickness $\delta$ [18]. The heat $Q_{b}$ extracted across the lower mantle thermal boundary layer is related to the heat $Q_{c}$ driving core convection by $Q_{c}=Q_{b}+Q_{h}$. Since $\left\langle R_{h}\right\rangle<0, Q_{h}<0$ and the reaction decreases $Q_{c}$ for a given $Q_{b}$, which lowers the core cooling rate and decreases the power available to the geodynamo $[3,25]$.

The flux of $\mathrm{O}$ from the solid mantle is estimated as $I_{\mathrm{O}} \sim$ $10^{3}-10^{4} \mathrm{~kg} \mathrm{~s}^{-1}$ [59], which gives $Q_{h} \sim 10^{-2}-10^{-1} \mathrm{TW}$, a negligible value. However, flux from the vigorously convecting magma ocean is likely to be much larger than from the relatively sluggish solid mantle. Thermal boundary layers in the magma ocean are perhaps only $1 \mathrm{~cm}$ thick [67], and chemical boundary layers are probably even thinner owing to the smaller compositional diffusion coefficient. Using $\delta=1 \mathrm{~cm}$, a compositional change of $\Delta c=1 \%$ across the boundary layer and other values above yields a flux of $8 \times 10^{8} \mathrm{~kg} \mathrm{~s}^{-1}$ and a heat sink of $Q_{h}=600 \mathrm{TW}$, while a value of $\delta=1 \mathrm{~m}$ reduces the flux and sink to $I_{\mathrm{O}}=8 \times 10^{6} \mathrm{~kg} \mathrm{~s}^{-1}$ and $Q_{h}=6 \mathrm{TW}$. The rather large uncertainties on $D, \Delta c$, and $\delta$ allow higher or lower estimates for $I_{\mathrm{O}}$ and $Q_{h}$ than those above, but values towards the lower end are more compatible with conventional estimates of CMB heat flow in early times $[3,68]$.

These high values of $Q_{h}$ are unlikely to be sustainable on long timescales because transfer of oxygen to the core would reduce the compositional difference across the lower mantle chemical boundary layer, and hence, $I_{\mathrm{O}}$. Indeed, if the top of the core becomes highly enriched in oxygen, then the mass flux of $\mathrm{FeO}$ may be limited by slow diffusion through the stratified upper core rather than by dynamics on the mantle side of the CMB. Realistic scenarios can be obtained only by solving conservation equations that couple $\mathrm{FeO}$ evolution in the mantle and core, which requires a separate study. Here we provide context for our estimates of $Q_{h}$ by comparing them to values of $Q_{b}$ during the early stages of core-mantle evolution.

Estimates of $Q_{b}=k \Delta T_{s} / \delta_{T}$ are uncertain because they depend on the superadiabatic temperature drop $\Delta T_{s}$ across the lower thermal boundary layer of thickness $\delta_{T}(k \sim$ $10 \mathrm{~W} \mathrm{~m}^{-1} \mathrm{~K}^{-1}$ the magma-ocean thermal conductivity [56]), $\Delta T_{s}$ is unknown but could be rather small if the magma ocean is vigorously convecting. Taking $\Delta T_{s}=$ $10^{-3} \mathrm{~K}$, which is similar to estimates for Earth's presentday core, and $\delta_{T}=1 \mathrm{~cm}$ as above suggests $Q_{b} \sim 100 \mathrm{TW}$, but uncertainties in the parameters could allow a factor of 10 variation either way. Core evolution models produce values of $Q_{b}$ ranging from 40 to $50 \mathrm{TW}$ [68] to well over 50 TW [69] around 4.0-4.5 Ga, though these models assume that the mantle is solid. A previous model of the basal magma ocean [56] produced low values of $Q_{b}<10 \mathrm{TW}$, though it was assumed that the core and mantle cooled at the same rate. In reality, $Q_{h}$ and $Q_{b}$ are coupled because heat released through the exothermic reaction will change the temperature at the top of the core, which in turn changes $\Delta T_{s}$. Nevertheless, the simple estimates above suggest that the effect is large enough to warrant further investigation, which could be accomplished 
by relaxing the constraint in existing core evolution models of no mass transfer between the core and mantle $[3,20,25]$.

\section{CONCLUSIONS}

The manner in which elements partition between silicates and metals at high pressure and temperature is critical for determining the structure, dynamics, and evolution of the terrestrial planets. The relative proportions of light elements in the core and mantle set during formation of the planet dictates the melting or freezing properties of these regions and the manner in which they flow, while partitioning of these elements during core freezing provides crucial power to generate planetary magnetic fields.

In this paper, we present three complementary and independent methods for calculating the chemical potentials of arbitrary solutes in solution. Excellent agreement is demonstrated by applying these techniques to study the partitioning of $\mathrm{FeO}$ between liquid metal and silicate melts at high pressure and temperature, providing crossvalidation and independent support. This work paves the way for establishing the partitioning behavior of all relevant elements and in particular silicon and magnesium. The methods allow calculations at pressures above $100 \mathrm{GPa}$ and temperatures of several thousand kelvin and therefore span the pressure-temperature conditions thought to have occurred during and following core formation in Earth and other terrestrial bodies.

Our $\mathrm{FeO}$ partitioning data show that oxygen strongly partitions into liquid metal at high pressure and temperature. These results suggest oxygen concentrations in the core during the latter stages of its formation that exceed present-day values, implying that the addition of oxygen at this time led to the formation of a stably stratified layer below the CMB. Future calculations are needed to establish whether this layer is related to anomalous seismic structure at the top of Earth's present-day core [70], in particular, whether the layer could resist disruption due to convection in the deeper core [59,71] or from the presence of thermal anomalies in the lower mantle [72]. We also show that heat released by the exothermic reaction could have limited the heat flow out of the core in early times. Reduced heat flow slows the core cooling rate, lowering the power available to the early dynamo, further exacerbating the energetic limitations imposed by high core thermal conductivity (e.g., Refs. $[3,25,63,73,74])$ and reinforcing the need for alternative power sources for the ancient geomagnetic field $[4,6]$. Future models of core thermochemical evolution should account for this effect when accessing the conditions that gave rise to Earth's ancient magnetic field.

\section{ACKNOWLEDGMENTS}

D. A. and M. P. acknowledge support from the Natural Environment Research Council (NERC) Grants No. NE/ M000990/1 and No. NE/R000425/1. C. D. acknowledges a
NERC personal fellowship, Grant No. NE/L011328/1. Calculations are performed on the Monsoon2 system, a collaborative facility supplied under the Joint Weather and Climate Research Programme, a strategic partnership between the UK Met Office and NERC. Calculations are also performed at University College London Research Computing on the Materials and Molecular Modelling hub Grant No. EP/P020194/1 and on the Oak Ridge Leadership Computing Facility (Contract No. DE-AC05-00OR22725). We also like to acknowledge the input of three anonymous referees.

\section{APPENDIX A}

We derive here the approximate relation Eq. (3), between constant pressure and constant volume variations. Let us consider two systems, 0 and 1 , with potential energies $U_{0}(\mathbf{R})$ and $U_{1}(\mathbf{R})$, respectively, and let us introduce the hybrid potential $U_{\lambda}(\mathbf{R})=\lambda U_{1}(\mathbf{R})+(1-\lambda) U_{0}(\mathbf{R})$. We are interested here in the quantity $\Delta F=F_{1}-F_{0}$ and its relation to $\Delta G=G_{1}-G_{0}$, where $F_{0}$ and $F_{1}$ are the Helmholtz free energies of the two systems at volume $V$ and temperature $T$, and $G_{0}$ and $G_{1}$ the corresponding Gibbs free energies at pressure $p$ and temperature $T$. In the following, we assume that the temperature is fixed, and so we do not display it explicitly. Noting that as we go from system 0 to system 1 , we have $d \lambda=1$, these free-energy differences can be written as

$$
\Delta F=\left(\frac{\partial F_{\lambda}}{\partial \lambda}\right)_{V}+\frac{1}{2}\left(\frac{\partial^{2} F_{\lambda}}{\partial \lambda^{2}}\right)_{V}+\ldots
$$

where $F_{\lambda}$ is the Helmholtz free energy of the system with potential energy $U_{\lambda}$, and the derivatives are taken at $\lambda=0$. Analogously, for the Gibbs free energy, we have

$$
\Delta G=\left(\frac{\partial G_{\lambda}}{\partial \lambda}\right)_{p}+\frac{1}{2}\left(\frac{\partial^{2} G_{\lambda}}{\partial \lambda^{2}}\right)_{p}+\ldots
$$

Since $G_{\lambda}=F_{\lambda}+p V$, we have

$$
\left(\frac{\partial G_{\lambda}}{\partial \lambda}\right)_{p}=\left(\frac{\partial F_{\lambda}}{\partial \lambda}\right)_{p}+p\left(\frac{\partial V}{\partial \lambda}\right)_{p}
$$

The differential of $F_{\lambda}$ with respect to variations of $V$ and $\lambda$ is

$$
d F_{\lambda}=\left(\frac{\partial F_{\lambda}}{\partial \lambda}\right)_{V} d \lambda+\left(\frac{\partial F_{\lambda}}{\partial V}\right)_{\lambda} d V
$$

and by writing $V=V(\lambda, p)$, we have $d V=[(\partial V) /$ $(\partial \lambda)]_{p} d \lambda+[(\partial V) /(\partial p)]_{\lambda} d p$, which once substituted in Eq. (A4) allows us to write the differential of $F_{\lambda}$ with respect to $\lambda$ and $p$, 


$$
\begin{aligned}
d F_{\lambda}= & \left(\frac{\partial F_{\lambda}}{\partial \lambda}\right)_{V} d \lambda+\left(\frac{\partial F_{\lambda}}{\partial V}\right)_{\lambda}\left[\left(\frac{\partial V}{\partial \lambda}\right)_{p} d \lambda+\left(\frac{\partial V}{\partial p}\right)_{\lambda} d p\right] \\
= & {\left[\left(\frac{\partial F_{\lambda}}{\partial \lambda}\right)_{V}+\left(\frac{\partial F_{\lambda}}{\partial V}\right)_{\lambda}\left(\frac{\partial V}{\partial \lambda}\right)_{p}\right] d \lambda } \\
& +\left(\frac{\partial F_{\lambda}}{\partial V}\right)_{\lambda}\left(\frac{\partial V}{\partial p}\right)_{\lambda} d p
\end{aligned}
$$

and so, considering $F=F(\lambda, p)$,

$$
\begin{aligned}
\left(\frac{\partial F_{\lambda}}{\partial \lambda}\right)_{p} & =\left(\frac{\partial F_{\lambda}}{\partial \lambda}\right)_{V}+\left(\frac{\partial F_{\lambda}}{\partial V}\right)_{\lambda}\left(\frac{\partial V}{\partial \lambda}\right)_{p} \\
& =\left(\frac{\partial F_{\lambda}}{\partial \lambda}\right)_{V}-p\left(\frac{\partial V}{\partial \lambda}\right)_{p}
\end{aligned}
$$

Comparing Eqs. (A3) and (A6), we have

$$
\left(\frac{\partial G_{\lambda}}{\partial \lambda}\right)_{p}=\left(\frac{\partial F_{\lambda}}{\partial \lambda}\right)_{V}
$$

This is nothing but a general thermodynamic equivalence between the derivative of $G$ at constant pressure and that of $F$ at constant volume with respect to some external perturbation, and indeed, it is the equivalence used in the definitions of the chemical potential in Eqs. (1) and (2) where the perturbation is due to the addition of one atom to the system. For finite differences, Eq. (A7) shows that variations of $G$ and $F$ are the same to linear order in the perturbation, and corrections appear only to higher orders. Since we compute the chemical potential as a finite difference $\mu\left(p, T, c_{X}\right)=\Delta G$ and $\mu\left(V, T, c_{X}\right)=\Delta F$, where $\Delta$ refers to a change caused by the addition of one atom of solute at constant pressure (volume) in the case of $\Delta G$ $(\Delta F)$, we have that to linear order $\mu\left(p, T, c_{X}\right)=$ $\mu\left(V, T, c_{X}\right)$. The perturbation causes a change of volume (pressure) if performed at constant pressure (volume), and therefore, the first correction in the equivalence between $\Delta G$ and $\Delta F$ is going to be quadratic with respect to the change of volume (pressure) as the atom is added at constant pressure (volume).

Let us now consider the second-order variations and define

$$
A_{\lambda}=\left(\frac{\partial G_{\lambda}}{\partial \lambda}\right)_{p}
$$

We have

$$
\left(\frac{\partial^{2} G_{\lambda}}{\partial \lambda^{2}}\right)_{p}=\left(\frac{\partial A_{\lambda}}{\partial \lambda}\right)_{p}
$$

The infinitesimal change of $A_{\lambda}$ with respect to variations of $V$ and $\lambda$ is

$$
d A_{\lambda}=\left(\frac{\partial A_{\lambda}}{\partial \lambda}\right)_{V} d \lambda+\left(\frac{\partial A_{\lambda}}{\partial V}\right)_{\lambda} d V
$$

and writing $A_{\lambda}=A_{\lambda}(\lambda, p)$, we obtain

$$
\left(\frac{\partial A_{\lambda}}{\partial \lambda}\right)_{p}=\left(\frac{\partial A_{\lambda}}{\partial \lambda}\right)_{V}+\left(\frac{\partial A_{\lambda}}{\partial V}\right)_{\lambda}\left(\frac{\partial V}{\partial \lambda}\right)_{p}
$$

Using [75]

$$
\left(\frac{\partial V}{\partial \lambda}\right)_{p}=-\left(\frac{\partial V}{\partial p}\right)_{\lambda}\left(\frac{\partial p}{\partial \lambda}\right)_{V}=\frac{V}{B_{T}}\left(\frac{\partial p}{\partial \lambda}\right)_{V}
$$

together with

$$
\left(\frac{\partial A_{\lambda}}{\partial V}\right)_{\lambda}=\left(\frac{\partial}{\partial V}\left[\frac{\partial F_{\lambda}}{\partial \lambda}\right]_{V}\right)_{\lambda}=\left(\frac{\partial}{\partial \lambda}\left[\frac{\partial F_{\lambda}}{\partial V}\right]_{\lambda}\right)_{V}=-\left(\frac{\partial p}{\partial \lambda}\right)_{V}
$$

we obtain

$$
\begin{aligned}
\left(\frac{\partial^{2} G_{\lambda}}{\partial \lambda^{2}}\right)_{p} & =\left(\frac{\partial A_{\lambda}}{\partial \lambda}\right)_{V}-\frac{V}{B_{T}}\left(\frac{\partial p}{\partial \lambda}\right)_{V}^{2} \\
& =\left(\frac{\partial^{2} F_{\lambda}}{\partial \lambda^{2}}\right)_{V}-\frac{V}{B_{T}} \delta p^{2}
\end{aligned}
$$

where $\delta p$ is the change of pressure as the potential energy is changed from $U_{0}$ to $U_{1}$ at constant volume $V, B_{T}=$ $-V[(\partial p) /(\partial V)]_{T}$, and we use $[(\partial p) /(\partial \lambda)]_{V}=\delta p$ valid to linear order in $\delta p$. We can now write our final expression for the relation of the change of Gibbs and Helmholtz free energies to second order:

$$
\Delta G=\Delta F-\frac{V}{2 B_{T}} \delta p^{2}+\ldots
$$

\section{APPENDIX B}

To keep the notation simpler, we work with the symmetric difference $F\left(V, T, N_{A}, N_{X}+d N\right)-F\left(V, T, N_{A}\right.$, $\left.N_{X}-d N\right)$. To obtain the asymmetric quantity $F\left(V, T, N_{A}\right.$, $\left.N_{X}\right)-F\left(V, T, N_{A}, N_{X}-d N\right)$ of Eq. (10), we then replace $d N \rightarrow d N / 2, \quad N_{X} \rightarrow N_{X}-d N / 2$, and $N \rightarrow N-d N / 2$. We have 


$$
\begin{aligned}
F\left(V, T, N_{A}, N_{X}\right. & +d N)-F\left(V, T, N_{A}, N_{X}-d N\right)-\Delta F=-k_{B} T \ln \frac{V^{2 d N}\left(N_{X}-d N\right) !}{\Lambda_{X}^{6 d N}\left(N_{X}+d N\right) !} \\
& =-k_{B} T \ln \frac{V^{2 d N}}{\Lambda_{X}^{6 d N} N^{2 d N}} \frac{N^{2 d N}\left(N_{X}-d N\right) !}{\left(N_{X}+d N\right) !}=-k_{B} T \ln \frac{V^{2 d N}}{\Lambda_{X}^{6 d N} N^{2 d N}}-k_{B} T \ln \frac{N^{2 d N}\left(N_{X}-d N\right) !}{\left(N_{X}+d N\right) !} \\
& =2 d N \mu_{X}^{\mathrm{PG}}(V, T, N)-k_{B} T \ln \frac{N^{2 d N}\left(N_{X}-d N\right) !}{\left(N_{X}+d N\right) !}
\end{aligned}
$$

with $\mu_{X}^{\mathrm{PG}}(V, T, N)$ the perfect gas chemical potential of $X$ in the system with temperature $T$. If $N_{X}-d N \gg 1$, we can rewrite the last term using the Stirling approximation:

$$
\begin{aligned}
& -k_{B} T \ln \frac{N^{2 d N}\left(N_{X}-d N\right) !}{\left(N_{X}+d N\right) !}=-k_{B} T \ln \frac{N^{2 d N} e^{2 d N}\left(N_{X}-d N\right)^{N_{X}-d N}}{\left(N_{X}+d N\right)^{N_{X}+d N}} \\
& =-k_{B} T\left\{2 d N[1+\ln N]+\left[\left(N_{X}-d N\right) \ln \left(N_{X}-d N\right)-\left(N_{X}+d N\right) \ln \left(N_{X}+d N\right)\right]\right\} \\
& \quad=-k_{B} T\left\{2 d N[1+\ln N]+N_{X}\left[\ln \left(N_{X}-d N\right)-\ln \left(N_{X}+d N\right)\right]-d N\left[\ln \left(N_{X}-d N\right)+\ln \left(N_{X}+d N\right)\right]\right\} .
\end{aligned}
$$

If we also have $d N \ll N_{X}$, we can expand the logarithms in Taylor series and retain only the terms linear in $d N / N_{X}$, which gives

$$
\begin{gathered}
F\left(V, T, N_{A}, N_{X}+d N\right)-F\left(V, T, N_{A}, N_{X}-d N\right) \\
\quad=2 d N\left[\mu_{X}^{\mathrm{PG}}(V, T, N)+k_{B} T \ln c_{X}\right]+\Delta F,
\end{gathered}
$$

where $c_{X}=N_{X} / N$. Equation (B7) is only valid in the thermodynamic limit. For finite values of $N_{X}$ or if $d N \simeq N_{X}$, the Stirling approximation and/or the Taylor expansion employed above are not accurate, and the error is given by the quantity

$$
\begin{aligned}
& \mathrm{FS}\left(N_{X}+d N, 2 d N\right) \\
& \quad=k_{B} T\left[\ln \frac{\left(N_{X}+d N\right) !}{N^{2 d N}\left(N_{X}-d N\right) !}-2 d N \log c_{X}\right] .
\end{aligned}
$$

We now refer back to Eq. (10), and with the substitutions $d N \rightarrow d N / 2, \quad N_{X} \rightarrow N_{X}-d N / 2$, and $N \rightarrow N-d N / 2$, and therefore also $v=V /(N-d N / 2)$, we obtain

$$
\begin{aligned}
& F\left(V, T, N_{A}, N_{X}\right)-F\left(V, T, N_{A}, N_{X}-d N\right) \\
& \quad=d N\left[\mu_{X}^{\mathrm{PG}}(v, T)+k_{B} T \ln c_{X}\right]+\mathrm{FS}\left(N_{X}, d N\right)+\Delta F,
\end{aligned}
$$

where now $c_{X}=\left(N_{X}-d N / 2\right) /(N-d N / 2)$ and $\Delta F$ is calculated as in Eq. (11).

[1] D. Rubie, F. Nimmo, and H. Melosh, Formation of Earth's Core, in Treatise on Geophysics, 2nd ed., edited by G. Schubert (Elsevier, Amsterdam, 2015), Vol. 9, pp. 43-79.
[2] S. Braginsky, Structure of the F Layer and Reasons for Convection in the Earth's Core, Sov. Phys. Dokl. 149, 8 (1963).

[3] F. Nimmo, Energetics of the Core, in Treatise on Geophysics, 2nd ed., edited by G. Schubert (Elsevier, Amsterdam, 2015), Vol. 8, pp. 27-55.

[4] J. G. O'Rourke and D. J. Stevenson, Powering Earth's Dynamo with Magnesium Precipitation from the Core, Nature (London) 529, 387 (2016).

[5] J. Badro, J. Siebert, and F. Nimmo, An Early Geodynamo Driven by Exsolution of Mantle Components from Earth's Core, Nature (London) 536, 326 (2016).

[6] K. Hirose, G. Morard, R. Sinmyo, K. Umemoto, J. Hernlund, G. Helffrich, and S. Labrosse, Crystallization of Silicon Dioxide and Compositional Evolution of the Earth's Core, Nature (London) 543, 99 (2017).

[7] G. Masters and D. Gubbins, On the Resolution of Density within the Earth, Phys. Earth Planet. Inter. 140, 159 (2003).

[8] D. Alfè, M. Gillan, and G. Price, Composition and Temperature of the Earth's Core Constrained by Combining Ab Initio Calculations and Seismic Data, Earth Planet. Sci. Lett. 195, 91 (2002).

[9] J. Badro, A. Côté, and J. Brodholt, A Seismologically Consistent Compositional Model of Earth's Core., Proc. Natl. Acad. Sci. U.S.A. 111, 7542 (2014).

[10] R. Deguen, M. Landeau, and P. Olson, Turbulent MetalSilicate Mixing, Fragmentation, and Equilibration in Magma Oceans, Earth Planet. Sci. Lett. 391, 274 (2014).

[11] D. C. Rubie, S. A. Jacobson, A. Morbidelli, D. P. O'Brien, E. D. Young, J. de Vries, F. Nimmo, H. Palme, and D. J. Frost, Accretion and Differentiation of the Terrestrial Planets with Implications for the Compositions of EarlyFormed Solar System Bodies and Accretion of Water, Icarus 248, 89 (2015).

[12] J. Badro, J. Brodholt, H. Piet, J. Siebert, and F. Ryerson, Core Formation and Core Composition from Coupled 
Geochemical and Geophysical Constraints, Proc. Natl. Acad. Sci. U.S.A. 112, 12310 (2015).

[13] Y. Asahara, D. J. Frost, and D. C. Rubie, Partitioning of $\mathrm{FeO}$ between Magnesiowustite and Liquid Iron at High Pressures and Temperatures: Implications for the Composition of the Earth's Outer Core, Earth Planet. Sci. Lett. 257, 435 (2007).

[14] H. Ozawa, K. Hirose, M. Mitome, Y. Bando, N. Sata, and Y. Ohishi, Chemical Equilibrium between Ferropericlase and Molten Iron to $134 \mathrm{GPa}$ and Implications for Iron Content at the Bottom of the Mantle, Geophys. Res. Lett. 35, L05308 (2008).

[15] D. J. Frost, Y. Asahara, D. C. Rubie, N. Miyajima, L. S. Dubrovinsky, C. Holzapfel, E. Ohtani, M. Miyahara, and T. Sakai, Partitioning of Oxygen between the Earth's Mantle and Core, J. Geophys. Res. 115, B02202 (2010).

[16] E. Knittle and R. Jeanloz, Earth's Core-Mantle Boundary: Results of Experiments at High Pressures and Temperatures, Science 251, 1438 (1991).

[17] N. Takafuji, K. Hirose, M. Mitome, and Y. Bando, Solubilities of $\mathrm{O}$ and $\mathrm{Si}$ in Liquid Iron in Equilibrium with $(\mathrm{Mg}, \mathrm{Fe}) \mathrm{SiO}_{3}$ Perovskite and the Light Elements in the Core, Geophys. Res. Lett. 32, L06313 (2005).

[18] C. Davies, M. Pozzo, D. Gubbins, and D. Alfè, Partitioning of Oxygen between Ferropericlase and Earth's Liquid Core, Geophys. Res. Lett. 45, 6042 (2018).

[19] J. Brodholt and J. Badro, Composition of the Low Seismic Velocity E' Layer at the Top of Earth's Core, Geophys. Res. Lett. 44, 8303 (2017).

[20] S. Labrosse, J. W. Hernlund, and K. Hirose, Fractional Melting and Freezing in the Deep Mantle and Implications for the Formation of a Basal Magma Ocean, in The Early Earth: Accretion and Differentiation, edited by J. Badro and M. Walter Geophysical Monograph. Vol. 212 (AGU, 2015), Chap. 7, pp. 123-142.

[21] J. Tarduno, R. D. Cottrell, M. K. Watkeys, A. Hofmann, P. V. Doubrovine, E. E. Mamajek, D. Liu, D. G. Sibeck, L. P. Neukirch, and Y. Usui, Geodynamo, Solar Wind, and Magnetopause 3.4 to 3.45 Billion Years Ago, Science 327, 1238 (2010).

[22] M. Pozzo, C. Davies, D. Gubbins, and D. Alfè, Thermal and Electrical Conductivity of Iron at Earth's Core Conditions, Nature (London) 485, 355 (2012).

[23] H. Gomi, K. Ohta, K. Hirose, S. Labrosse, R. Caracas, M. J. Verstraete, and J. W. Hernlund, The High Conductivity of Iron and Thermal Evolution of the Earth's Core, Phys. Earth Planet. Inter. 224, 88 (2013).

[24] K. Ohta, Y. Kuwayama, K. Hirose, K. Shimizu, and Y. Ohishi, Experimental Determination of the Electrical Resistivity of Iron at Earth's Core Conditions, Nature (London) 534, 95 (2016).

[25] C. J. Davies, Cooling History of Earth's Core with High Thermal Conductivity, Phys. Earth Planet. Inter. 247, 65 (2015).

[26] Z. Konôpková, R. McWilliams, N. Gómez-Pérez, and A. Goncharov, Direct Measurement of Thermal Conductivity in Solid Iron at Planetary Core Conditions, Nature (London) 534, 99 (2016).

[27] J. Xu, P. Zhang, K. Haule, J. Minar, S. Wimmer, H. Ebert, and R.E. Cohen, Thermal Conductivity and Electrical
Resistivity of Solid Iron at Earth's Core Conditions from First Principles, Phys. Rev. Lett. 121, 096601 (2018).

[28] F. Nimmo, G. Price, J. Brodholt, and D. Gubbins, The Influence of Potassium on Core and Geodynamo Evolution, Geophys. J. Int. 156, 363 (2004).

[29] R. A. Fischer, Y. Nakajima, A. J. Campbell, D. J. Frost, D. Harries, F. Langenhorst, N. Miyajima, K. Pollok, and D. C. Rubie, High Pressure Metal-Silicate Partitioning of $\mathrm{Ni}, \mathrm{Co}, \mathrm{V}, \mathrm{Cr}$, Si, and $\mathrm{O}$, Geochim. Cosmochim. Acta 167, 177 (2015).

[30] J. Siebert, J. Badro, D. Antonangeli, and F. J. Ryerson, Metal-Silicate Partitioning of Ni and Co in a Deep Magma Ocean, Earth Planet. Sci. Lett. 321, 189 (2012).

[31] M. Bouhifd and A. Jephcoat, Convergence of Ni and Co Metal-Silicate Partition Coefficients in the Deep MagmaOcean and Coupled Silicon-Oxygen Solubility in Iron Melts at High Pressures, Earth Planet. Sci. Lett. 307, 341 (2011).

[32] T.-A. Suer, J. Siebert, L. Remusat, N. Menguy, and G. A. Fiquet, Sulfur-Poor Terrestrial Core Inferred from MetalSilicate Partitioning Experiments, Earth Planet. Sci. Lett. 469, 84 (2017).

[33] K. Tsuno, D. J. Frost, and D. C. Rubie, Simultaneous Partitioning of Silicon and Oxygen into the Earth's Core during Early Earth Differentiation, Geophys. Res. Lett. 40, 66 (2013).

[34] B. A. Chidester, Z. Rahman, K. Righter, and A. J. Campbell, Metal-Silicate Partitioning of U: Implications for the Heat Budget of the Core and Evidence for Reduced $U$ in the Mantle, Geochim. Cosmochim. Acta 199, 1 (2017).

[35] D. Andrault, S. Petitgirard, G. L. Nigro, J.-L. Devidal, G. Veronesi, G. Garbarino, and M. Mezouar, Solid-Liquid Iron Partitioning in Earth's Deep Mantle, Nature (London) 487, 354 (2012).

[36] P. Hohenberg and W. Kohn, Inhomogeneous Electron Gas, Phys. Rev. 136, B864 (1964).

[37] W. Kohn and L. Sham, Self-Consistent Equations Including Exchange and Correlation Effects, Phys. Rev. 140, A1133 (1965).

[38] D. Alfè, G. D. Price, and M. J. Gillan, Ab Initio Chemical Potentials of Solid and Liquid Solutions and the Chemistry of the Earth's Core, J. Chem. Phys. 116, 7127 (2002).

[39] F. Mandle, Statistical Physics, 2nd ed. (J. Wiley and Sons, Plymouth, 1988), p. 311.

[40] Y. Wang and J. P. Perdew, Correlation Hole of the SpinPolarized Electron Gas, with Exact Small-Wave-Vector and High-Density Scaling, Phys. Rev. B 44, 13298 (1991).

[41] L. Stixrude, R. E. Cohen, and D. J. Singh, Iron at High Pressure: Linearized-Augmented-Plane-Wave Computations in the Generalized-Gradient Approximation, Phys. Rev. B 50, 6442 (1994).

[42] P. Söderlind, J. A. Moriarty, and J. M. Wills, First-Principles Theory of Iron up to Earth-Core Pressures: Structural, Vibrational, and Elastic Properties, Phys. Rev. B 53, 14063 (1996).

[43] D. Alfè, G. Kresse, and M. J. Gillan, Structure and Dynamics of Liquid Iron under Earth's Core Conditions, Phys. Rev. B 61, 132 (2000).

[44] H. K. Mao et al., Phonon Density of States of Iron Up to 153 GPa, Science 292, 914 (2001). 
[45] D. Alfè, G. D. Price, and M. J. Gillan, Iron under Earth's Core Conditions: Liquid-State Thermodynamics and HighPressure Melting Curve from Ab Initio Calculations, Phys. Rev. B 65, 165118 (2002).

[46] M. Pozzo, C. Davies, D. Gubbins, and D. Alfè, Transport Properties for Liquid Silicon-Oxygen-Iron Mixtures at Earth's Core Conditions, Phys. Rev. B 87, 014110 (2013).

[47] D. Alfè, M. J. Gillan, and G. D. Price, Constraints on the Composition of the Earth's Core from Ab-Initio Calculations, Nature (London) 405, 172 (2000).

[48] E. S. Posner, D. C. Rubie, D. J. Frost, and G. SteinleNeumann, Experimental Determination of Oxygen Diffusion in Liquid Iron at High Pressure, Earth Planet. Sci. Lett. 464, 116 (2017).

[49] G. Kresse and J. Furthmüller, Efficient Iterative Schemes for Ab Initio Total-Energy Calculations Using a Plane-Wave Basis Set, Phys. Rev. B 54, 11169 (1996).

[50] P. E. Blöchl, Projector Augmented-Wave Method, Phys. Rev. B 50, 17953 (1994).

[51] G. Kresse and D. Joubert, From Ultrasoft Pseudopotentials to the Projector Augmented-Wave Method, Phys. Rev. B 59, 1758 (1999).

[52] D. Alfè, Ab Initio Molecular Dynamics, a Simple Algorithm for Charge Extrapolation, Comput. Phys. Commun. 118, 31 (1999).

[53] S. Nosé, A Molecular Dynamics Method for Simulations in the Canonical Ensemble, Mol. Phys. 52, 255 (1984); A Unified Formulation of the Constant Temperature Molecular Dynamics Methods, J. Chem. Phys. 81, 511 (1984).

[54] Z. Du, C. Jackson, N. Bennett, P. Driscoll, J. Deng, K. K. M. Lee, E. Greenberg, V. B. Prakapenka, and Y. Fei, Insufficient Energy from $\mathrm{MgO}$ Exsolution to Power Early Geodynamo, Geophys. Res. Lett. 44, 11,376 (2017).

[55] R. Nomura, H. Ozawa, S. Tateno, K. Hirose, J. Hernlund, S. Muto, H. Ishii, and N. Hiraoka, Spin Crossover and IronRich Silicate Melt in the Earth's Deep Mantle, Nature (London) 473, 199 (2011).

[56] S. Labrosse, J. Hernlund, and N. Coltice, A Crystallizing Dense Magma Ocean at the Base of the Earth's Mantle, Nature (London) 450, 866 (2007).

[57] C. Liebske and D. J. Frost, Melting Phase Relations in the $\mathrm{MgO}-\mathrm{MgSiO}_{3}$ System between 16 and 26 GPa: Implications for Melting in Earth's Deep Interior, Earth Planet. Sci. Lett. 345, 159 (2012).

[58] E. Garnero, A. McNamara, and S.-H. Shim, Continent-Sized Anomalous Zones with Low Seismic Velocity at the Base of Earth's Mantle, Nat. Geosci. 9, 481 (2016).

[59] B. Buffett and C. Seagle, Stratification of the Top of the Core due to Chemical Interactions with the Mantle, J. Geophys. Res. 115, B04407 (2010).

[60] S. Glane and B. Buffett, Enhanced Core-Mantle Coupling due to Stratification at the Top of the Core, Front. Earth Sci. 6, 171 (2018).
[61] B. Buffett, Geomagnetic Fluctuations Reveal Stable Stratification at the Top of the Earth's Core, Nature (London) 507, 484 (2014).

[62] S. I. Braginsky, MAC-Oscillations of the Hidden Ocean of the Core, J. Geomagn. Geoelectr. 45, 1517 (1993).

[63] C. Davies, M. Pozzo, D. Gubbins, and D. Alfè, Constraints from Material Properties on the Dynamics and Evolution of Earth's Core, Nat. Geosci. 8, 678 (2015).

[64] S. A. Jacobson, D. C. Rubie, J. Hernlund, A. Morbidelli, and M. Nakajima, Formation, Stratification, and Mixing of the Cores of Earth and Venus, Earth Planet. Sci. Lett. 474, 375 (2017).

[65] M. Landeau, P. Olson, R. Deguen, and B. H. Hirsh, Core Merging and Stratification Following Giant Impact, Nat. Geosci. 9, 786 (2016).

[66] D. Gubbins, D. Alfè, G. Masters, G. Price, and M. Gillan, Gross Thermodynamics of Two-Component Core Convection, Geophys. J. Int. 157, 1407 (2004).

[67] V. Solomatov, Magma Oceans and Primordial Mantle Differentiation, in Treatise on Geophysics, 2nd ed., edited by G. Schubert (Elsevier, Amsterdam, 2015), Vol. 10, pp. 81-104.

[68] P. Driscoll and D. Bercovici, On the Thermal and Magnetic Histories of Earth and Venus: Influences of Melting, Radioactivity, and Conductivity, Phys. Earth Planet. Inter. 236, 36 (2014).

[69] T. Nakagawa and P. Tackley, Influence of Combined Primordial Layering and Recycled MORB on the Coupled Thermal Evolution of Earth's Mantle and Core, Geochem. Geophys. Geosys. 15, 619 (2014).

[70] G. Helffrich and S. Kaneshima, Outer-Core Compositional Stratification from Observed Core Wave Speed Profiles, Nature (London) 468, 807 (2010).

[71] D. Gubbins and C. Davies, The Stratified Layer at the CoreMantle Boundary Caused by Barodiffusion of Oxygen, Sulphur and Silicon, Phys. Earth Planet. Inter. 215, 21 (2013).

[72] G. A. Cox, C. J. Davies, P. W. Livermore, and J. Singleton, Penetration of Boundary-Driven Flows into a Rotating Spherical Thermally Stratified Fluid, J. Fluid Mech. 864, 519 (2019).

[73] S. Labrosse, Thermal Evolution of the Core with a High Thermal Conductivity, Phys. Earth Planet. Inter. 247, 36 (2015).

[74] D. Gubbins, D. Alfè, C. Davies, and M. Pozzo, On Core Convection and the Geodynamo: Effects of High Electrical and Thermal Conductivity, Phys. Earth Planet Inter. 247, 56 (2015).

[75] Given the function $V(\lambda, p)$, its differential is $d V=$ $\left[(\partial V) /(\partial \lambda)_{p} d \lambda+[(\partial V / \partial p)]_{\lambda} d p\right.$. For $d V=0$, we have $[(\partial V / \partial p)]_{\lambda} d p=-[(\partial V / \partial \lambda)]_{p} d \lambda$, and therefore, $[(\partial V / \partial \lambda)]_{p}=-[(\partial V / \partial p)]_{\lambda}[(\partial p / \partial \lambda)]_{V}$. 